Chronique des activités archéologiques de

\title{
Kvarner (Croatie)
}

Prospection-inventaire des sites ecclésiaux et monastiques : campagne 2013

Morana Čaušević-Bully et Sébastien Bully

\section{(2) OpenEdition}

Journals

Édition électronique

URL : http://journals.openedition.org/cefr/1059

DOI : $10.4000 /$ cefr. 1059

ISSN : 2282-5703

Éditeur

École française de Rome

Référence électronique

Morana Čaušević-Bully et Sébastien Bully, « Kvarner (Croatie) », Chronique des activités archéologiques de l'École française de Rome [En ligne], Balkans, mis en ligne le 14 janvier 2014, consulté le 23 mars 2020. URL : http://journals.openedition.org/cefr/1059; DOI : https://doi.org/10.4000/cefr.1059

Ce document a été généré automatiquement le 23 mars 2020.

(c) École française de Rome 


\section{Kvarner (Croatie)}

Prospection-inventaire des sites ecclésiaux et monastiques : campagne 2013

\section{Morana Čaušević-Bully et Sébastien Bully}

\section{NOTE DE L'ÉDITEUR}

Avec la collaboration de Ivan Valent, Laurent Fiocchi, et les contributions de Luka Babić, Josip Burmaz, Pascale Chevalier et Miro Vuković

1 Le programme de prospection-inventaire des sites ecclésiaux insulaires engagé en $2010^{1}$ est structuré autour de trois axes majeurs:

Identification des sites monastiques potentiels à partir des sources écrites, des données archéologiques, architecturales et topographiques ;

Conditions et modalités de l'installation et de la diffusion du monachisme insulaire dans le Kvarner, entre le ${ }_{\mathrm{V}}{ }_{\mathrm{e}}^{\mathrm{e}}$ et le $\mathrm{XI}_{\mathrm{XI}}{ }^{\mathrm{e}}$ siècle : occupation du sol et voies maritimes, construction de l'espace ;

Topographie monastique et architecture cultuelle : héritages et influences, cénobitisme et érémitisme.

2 Parmi les six sites retenus au terme de la première campagne menée en $2010^{2}$, nous sommes intervenus en 2013 sur quatre d'entre eux : le site de l'îlot de Lukovac (île de Rab), l'église des Saints-Jean-Pantaléon (îlot d'Oruda), les complexes de Martinšćića (île de Cres) et de Mirine-Fulfinum (île de Krk) (fig. 1). 
Fig. 1 - Carte de l'archipel du Kvarner et localisation des sites étudiés.

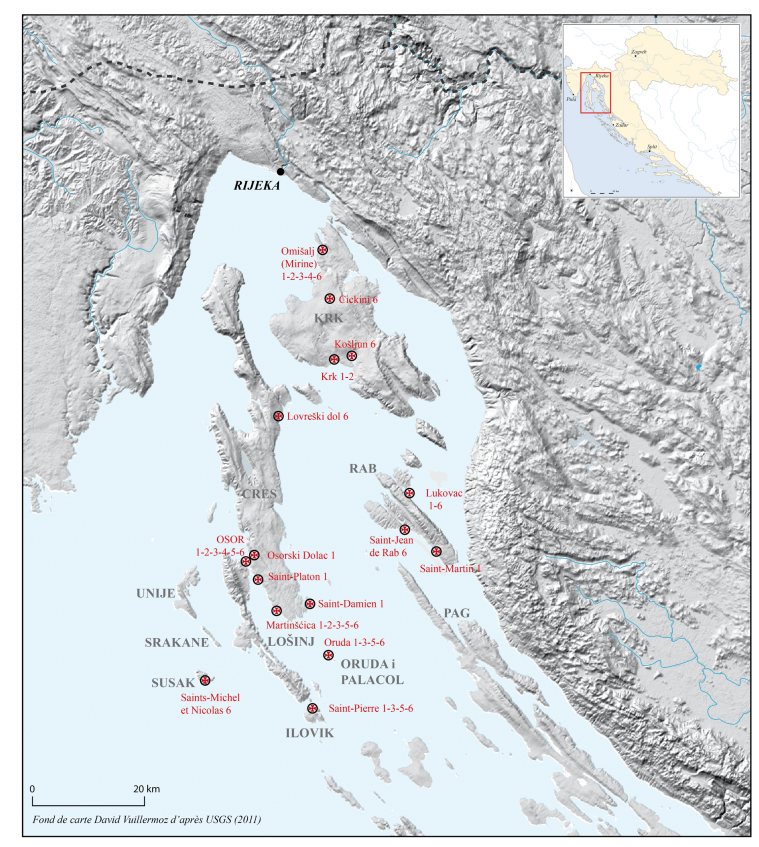

Pl. 1 - Archipel du Kvarner, carte des sites étudiés entre 2010 et 2013

(i) Sites étudiés

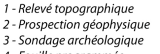

4- Fouille programmée
5-Etude d'archéologie du bâti

D’après M. Čaušević-Bully et S. Bully.

3 La campagne s'est déroulée en deux temps : du 15 avril au 27 avril et du 2 au 12 juillet ${ }^{3}$. Les interventions ont produit une documentation inédite à travers des relevés topographiques, des relevés d'élévations par scanner 3D, des restitutions d'élévations en 3D, des sondages ou l'engagement de fouille archéologique. L'ensemble des interventions participe à l'évaluation du potentiel archéologique enfoui ou en élévation des sites afin d'en préciser la datation et la nature potentiellement monastique.

\section{Martinšćica, île de Cres (troisième campagne) : sondages dans le complexe architectural et étude des élévations de la grande église paléochrétienne}

Lors de cette troisième opération sur le complexe de Martinšćica, nous avons poursuivi sa documentation archéologique et l'évaluation de son potentiel archéologique par l'ouverture de deux sondages sur deux secteurs clés pour la connaissance du site et sa datation. Le premier sondage a porté sur un des tas d'épierrement reconnu en prospection comme un possible bâtiment (bâtiment L), puis le second sur une construction de plan centré ou partiellement centré désigné comme " Martinšćica 2 » (fig. 2). 
Fig. 2 - Martinšćica, plan masse du complexe, de l'église, et localisation des sondages 2013.

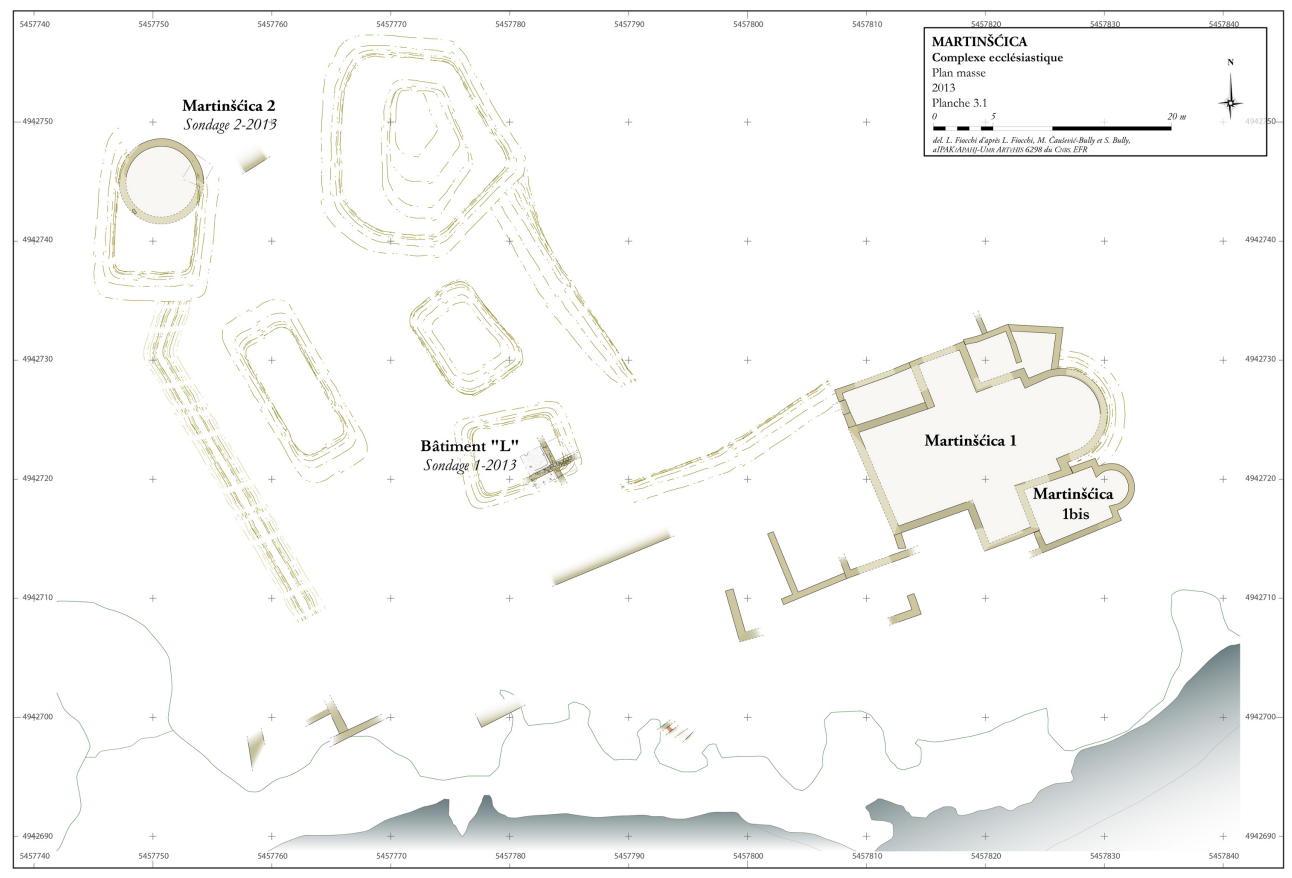

Infographie et plan L. Fiocchi, d'après M. Čaušević-Bully et S. Bully.

5 La grande église du complexe - Martinšćica 1 - est datée habituellement de la période justinienne, ou légèrement plus tardivement, du VII siècle $^{4}$. Dans nos travaux des années précédentes, nous avons pu démontrer que cette grande église de plan en croix grecque est consécutive de plusieurs phases de construction. En effet, l'analyse des maçonneries du mur gouttereau nord et du transept nord a révélé une phase primitive à la grande église, tandis que la chapelle latérale sud (Martinšćica 1bis) et les annexes sont postérieures. À partir de ce constat, se pose la question de la datation et de la nature de ou des phases de construction qui précédent celle de la grande église, et du maintien ou du changement de la fonction même du complexe.

\section{Sondage 1 - Bâtiment L}

Le sondage 1 (dimensions : 3,09 x 3,20 m) a été ouvert à l'emplacement du Bâtiment L où une arase de mur était visible en surface. Il a révélé des maçonneries perpendiculaires délimitant quatre salles différentes - enregistrées comme L1, L2, L3 ${ }^{5}$ et L4 - appartenant à trois états de construction (fig. 3). 
Fig. 3 - Martinšćica, bâtiment L, relevé du sondage.

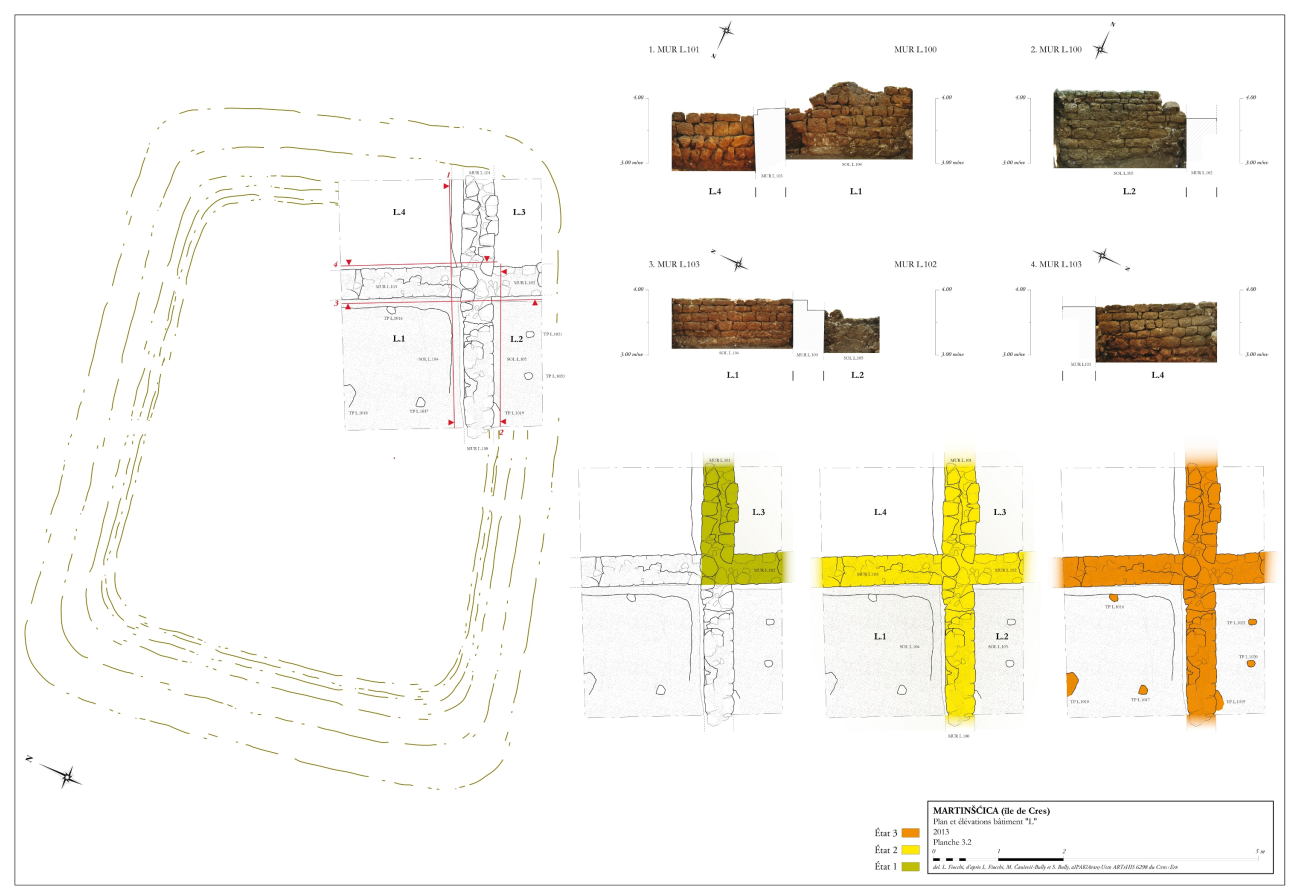

Infographie et plan L. Fiocchi, d'après M. Čaušević-Bully et S. Bully.

7 Les sols et les maçonneries du bâtiment sont scellés par des couches de démolition et par l'humus (us 1000) entre les cotes 3,53 et 2,79 $\mathrm{m} / \mathrm{nv}$. La première couche reconnue sous l'humus (us 1001) recouvrait l'ensemble de quatre pièces. Elle se caractérise par sa couleur foncée, presque noire, organique. Son mobilier est hétérogène : fragments de tegulae de divers modèles, imbrices, fragments de briques (d'une épaisseur importante d'environ 6,5 cm), un fragment de mortier en calcaire, des coquillages, de la céramique commune, des nodules d'argile d'un clayonnage ou d'un sol. Cette couche peu épaisse (d'une dizaine de centimètres) recouvrait directement une couche de démolition (us 1002) commune à toutes les pièces et observée sur l'ensemble de la surface du sondage. Elle est principalement composée de moellons calcaires provenant des maçonneries, noyés dans de la terre noire organique infiltrée de la couche supérieure (us 1001). Ce niveau contient un mobilier très similaire à celui trouvé dans la couche supérieure.

Sous la couche de démolition 1002 commune à l'ensemble du bâtiment L, chaque salle présente une séquence stratigraphique propre.

\section{Données stratigraphiques}

\section{Pièce L1 (fig. 4)}

Dans la pièce L1, limitée par les murs L.100 et L.103, le niveau de démolition 1002 recouvrait la couche 1004 qui se présentait comme un niveau rouge, argileux et très compact avec inclusion de petits nodules de mortier - côte $\pm 2,60 \mathrm{~m} / \mathrm{nv}$. 
Fig. 4 - Martinšćica, bâtiment L, sondage, vue de la pièce L1.

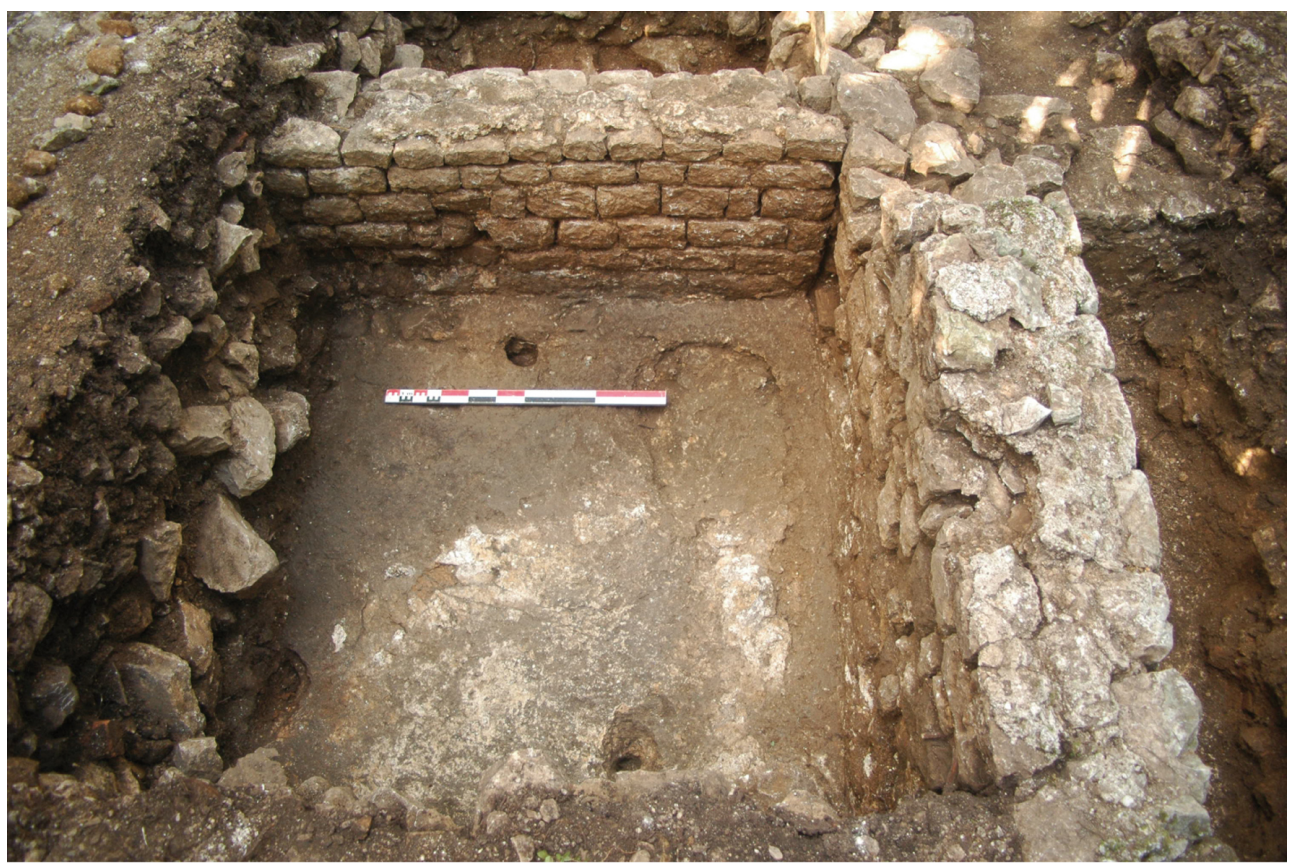

Cl. M. Čaušević-Bully.

10 À l'intérieur de cette couche, nous avons observé une lentille de terre plus organique noire et fine (1006), qui couvrait directement le sol 104. La couche 1006 contenait quelques escargots de mer (murex), des ossements de faune, des tessons de céramique rouge et d'amphores, de la gobeleterie ainsi que du verre à vitre concave (cive ?) ${ }^{6}$. La couche argileuse 1004 recouvrait une couche de terre très organique contenant des moellons non taillés (1005). Son mobilier est abondant en céramique (38 tessons), en gobeleterie et en verre à vitre (17 fragments). On notera la présence d'un fragment de tubulure et de quelques fragments de tegulae. La couche 1005 scellait la dernière phase d'occupation de la pièce reconnue par les niveaux 1008, 1009 et 1010. La couche 1008 était de faible épaisseur (sommet à $2,31 \mathrm{~m} / \mathrm{nv}$ ), noire et cendreuse, et elle contenait très peu de mobilier : quelques tessons de céramique d'assez bonne qualité, noire avec des inclusions de quartz ( $\mathrm{V}^{\mathrm{e}}-\mathrm{VII}{ }^{\mathrm{e}} \mathrm{s}$. ?), un fragment de verre et un os de faune. Le niveau 1008 couvrait une couche $(1009$, sommet à $2,29 \mathrm{~m} / \mathrm{nv})$ de mortier désagrégé pouvant résulter de la chute des enduits muraux. Celle-ci recouvrait directement le sol 104 de la pièce L1 (entre 2,18 - 2,23 $\mathrm{m} / \mathrm{nv}$ ).

11 Le sol 104 présente des traces d'une utilisation prolongée avec notamment un ragréage de mortier très compact (1012) qui a livré une monnaie en bronze (diamètre 13-14 mm) émise durant la période des fils de Constantin, entre 336 et $342^{7}$.

12 Le sol 104 et son ragréage 1012 sont percés par trois trous de piquets et de poteau $(1016,1017$ et 1018) appartenant à une structure en matériau périssable à l'intérieur de la pièce L1, à moins qu'il ne s'agisse d'une phase d'occupation tardive dans les ruines ( ?) de la salle L1. Le premier (1016), le long du mur 103, avait une forme grossièrement circulaire de $13 \mathrm{~cm}$ de diamètre par $\pm 20 \mathrm{~cm}$ de profondeur sous la surface du sol L.104. Le second trou de piquet (1017) était situé près du mur L.100. Son diamètre était de $7 \mathrm{~cm}$ pour une profondeur de $12 \mathrm{~cm}$, mais l'impact du percement dans le sol s'est traduit par une dépression superficielle beaucoup plus large, d'environ $19 \mathrm{~cm}$. Le 
troisième trou de poteau (1018), à la limite septentrionale du sondage, était légèrement plus important que les précédents avec $19 \mathrm{~cm}$ de diamètre pour une profondeur de $11 \mathrm{~cm}$. Son comblement contenait un fragment de céramique et un fragment de coquillage.

\section{Pièce L2 (fig. 5)}

Dans la pièce L2, délimitée par les murs 100 au nord et 102 à l'est, la séquence stratigraphique était plus pauvre.

Fig. 5 - Martinšćica, bâtiment L, sondage, vue de la pièce $L 2$.

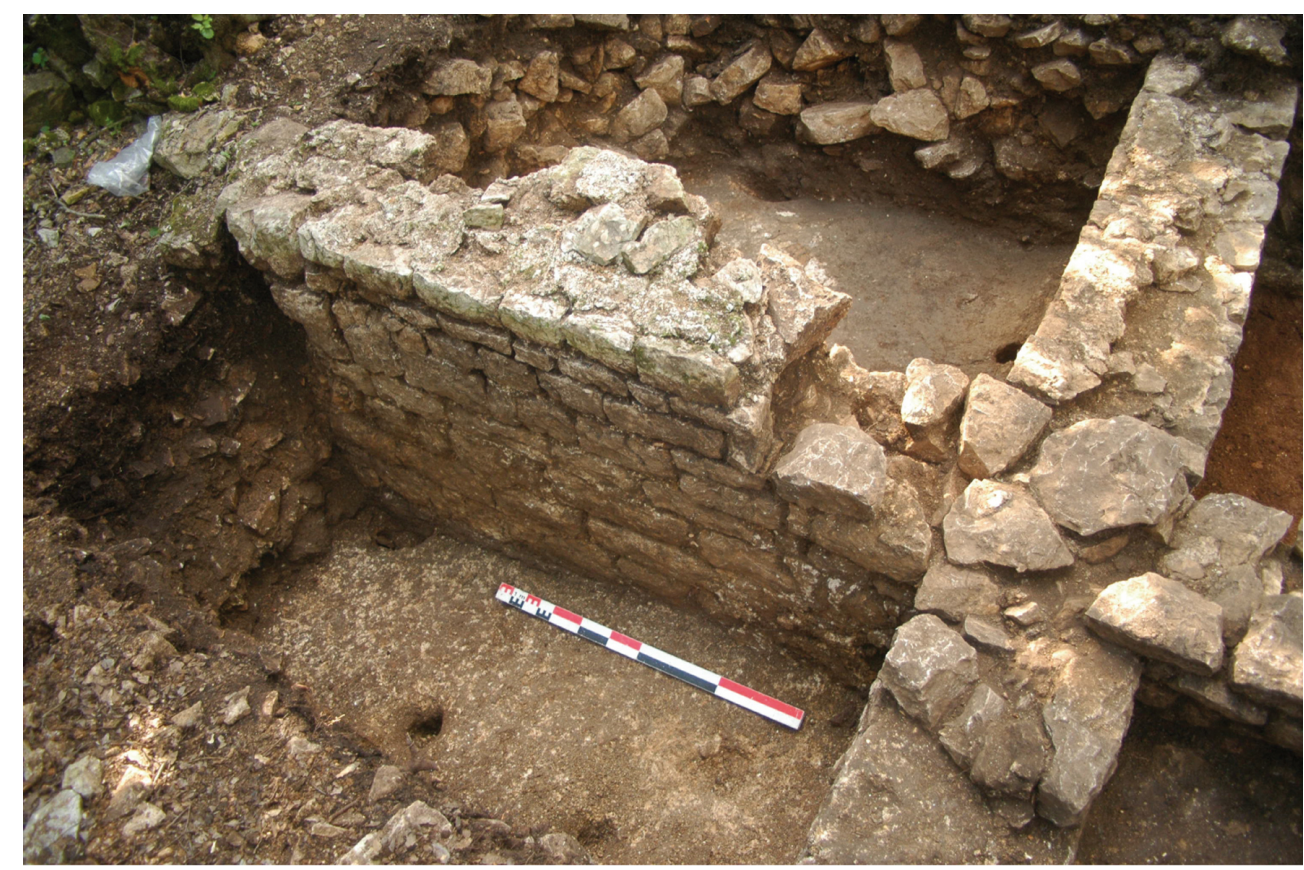

Cl. M. Čaušević-Bully.

Sous la dernière couche de démolition (1002) commune à toutes les pièces reconnues dans le sondage, on trouve un second niveau de démolition formée de gros moellons non taillés et des fragments de TCA, mélangés avec de l'argile très compacte de couleur jaune et des nodules du mortier (1011). Cette épaisse couche en recouvrait une plus fine, de terre (1013), couvrant directement sur le sol de la pièce L2 (105). Ce niveau d'occupation a livré un mobilier assez abondant, avec des restes d'huitres, une anse d'amphore de couleur ocre et d'engobe blanchâtre, des tessons de céramique réductrice tournée à fin dégraissant de quartz ou de calcaire. La céramique est décorée de vaguelettes irrégulières ou d'un fond peigné que l'on peut dater du V $\mathrm{V}^{\mathrm{e}} .^{8} \mathrm{Ce}$ mobilier est associé à la découverte d'une petite monnaie en bronze (diamètre $10 \mathrm{~mm}$ ) datable de la fin du IV $\mathrm{IV}^{\mathrm{e}}$., ou du début du $\mathrm{V}^{\mathrm{e}} \mathrm{s}$. (cf. fig. 2). Son mauvais état de conservation permet juste de distinguer un buste avec un diadème, une cape et un bouclier à droite sur l'avers. Le revers est illisible. La couche 1013 a également fourni des fragments de TCA. Outre un grand nombre de tegulae, il s'agit de deux types de briques - d'une épaisseur de $6 \mathrm{~cm}$ et de $10 \mathrm{~cm}$ - et de fragments de spicae d'un opus spicatum $-1,5 \mathrm{~cm}$ d'épaisseur par 5,7 cm de largeur. Le sol 105 était en mortier d'assez bonne qualité, situé entre les cotes $2,02 \mathrm{~m} / \mathrm{nv}$ (au milieu de la pièce) et 2,27 m/nv (près de l'angle créé 
par les murs 102 et 100). Tout comme la surface du sol 104, celui-ci est également percé de trois autres trous de piquets $(1019,1020$ et 1021). Un grand trou de piquet (1019) était percé contre le mur 100. Par sa forme ovoïdale, il ressemble fortement au trou 1018 de la pièce L1. Son fond est formé par la fondation débordante du mur 100 qui est à cet endroit à la côte $2,06 \mathrm{~m} / \mathrm{nv}$. Deux autres trous de piquets ont été repérés à une distance égale de mur 100, présageant l'existence d'un appentis le long de sa face méridionale. Les fonds de ces deux trous de piquets sont à la côte de $2,06 \mathrm{~m} / \mathrm{nv}$ pour le 1021 et à $2,02 \mathrm{~m} / \mathrm{nv}$ pour le 1020 .

\section{Pièce L4}

15 La stratigraphie de la pièce L4 est très simple et couvre le terrain naturel. Sous la couche 1002 on trouve une couche de terre rouge-orange mêlée à de gros moellons non taillés (1007). Le mobilier est principalement constitué de matériaux de construction (tegulae, nodules de mortier), avec cependant quelques tessons de céramique - dont un fragment d'amphore et plusieurs fragments d'une même poterie. On notera également la présence de scories ferreuses et de clous, ainsi que de quelques coquillages et d'ossements de faune. Cette couche recouvrait un lit de petites dalles calcaires directement posées à plat sur le sol géologique argileux et formant un possible niveau de circulation.

\section{Les maçonneries}

\section{Mur 100}

Son parement nord est préservé sur sept assises. L'épaisseur de mur est de 0,45 m. Les deux premières assises inférieures sont encore couvertes par un enduit blanchâtre (1023) en très mauvais état. Les assises de cette face sont assez irrégulières. La jonction avec le mur 103 est assurée par des moellons grossièrement taillés, disposés à sec (1022), en obturation d'une échancrure dans le mur de 0,40 m de largeur au sommet par $0,24 \mathrm{~m}$ au niveau du sol 104. Deux tuiles canal sont présentes dans l'échancrure dissimulée par le parement grossier 1022.

La face méridionale du mur 100 est préservée sur huit assises. Les assises de moellons de taille moyenne sont plus régulières, plus hautes dans la partie basse du mur. Les moellons sont liés par un liant mortier assez grossier de couleur jaune blanchâtre, avec une charge importante de gravillons de mer. Le mur 100 est postérieur au mur 102 contre lequel il s'adosse. Il porte les traces d'une seule couche d'enduit (1025), qui est identique à la deuxième couche d'enduit reconnue sur le parement ouest de mur 102. La fondation de ce mur n'est pas visible car elle est couverte entièrement par le sol 105. En revanche, grâce au percement du trou de piquet 1019, nous connaissons sa côte altimétrique qui est à $2.06 \mathrm{~m} / \mathrm{nv}$.

\section{Mur 101}

Il est préservé sur seulement deux assises d'élévation reposant sur une fondation formée de trois assises de moellons non taillés, saillants de $14 \mathrm{~cm}$ par rapport à l'élévation. Le sommet de la fondation se situe à la côte altimétrique $2,65 \mathrm{~m} / \mathrm{nv}$, soit $31 \mathrm{~cm}$ au-dessus du sommet de la fondation du mur 100 et $0,59 \mathrm{~m}$ au-dessus de la 
fondation du mur 102. La première assise de son élévation est composée de blocs taillés de forme carrée, tandis que la seconde assise est construite en petits moellons allongés.

\section{Mur 102}

19 Seul le parement ouest du mur a pu être observé, car la pièce L3 n'a pas été fouillée. Le mur est épais de $0,46 \mathrm{~m}$; les assises ne sont presque pas visibles, en raison du bon état de conservation des enduits. Une première couche d'enduit (1024) en fin mortier blanc recouvre uniquement le mur 102. Une seconde couche d'enduit (1025), plus grossière et de couleur jaune blanchâtre, a été appliquée sur la couche 1024 du mur 102, mais également sur la face sud du mur 100. Cette seconde couche laisse place dans la partie basse du parement (à $22 \mathrm{~cm}$ au-dessus du sol 105) à un autre enduit, plus fin et de couleur gris clair (1026).

\section{Mur 103}

Large de $0,47 \mathrm{~m}$, il est préservé sur $0,75 \mathrm{~m}$ de hauteur, sur 6 assises régulières, formées de moellons équarris allongés (pour le parement ouest). Les moellons sont liés par un mortier assez fin de couleur gris-blanc. Le liant est plus fin que celui du mur 100. Sa fondation (1015) est saillante par rapport à l'élévation de $13 \mathrm{~cm}$ et se situe à la côte $2,34 \mathrm{~m} / \mathrm{nv}$. Du côté du parement oriental, le mur est préservé sur $0,71 \mathrm{~m}$ de hauteur et cinq assises de d'élévation. La fondation est composée de blocs irréguliers sur deux assises. Elle est saillante par rapport au parement de $8 \mathrm{~cm}$ au maximum du côté nord et de seulement $2 \mathrm{~cm}$ du côté sud en raison de la réutilisation de la fondation du mur 101.

\section{Sondage 2 - Martinšćica 2 (espace VII) (fig. 6)}

Le sondage 2 (dimensions : 2,20 x 1,80 m) a été ouvert à l'emplacement de la jonction présumée entre l'abside (?) de Martinšćica 2 et un hypothétique mur d'épaulement. 
Fig. 6 - Martinšćica, vue générale du bâtiment de plan centré ( ?) Martinšćica 2.

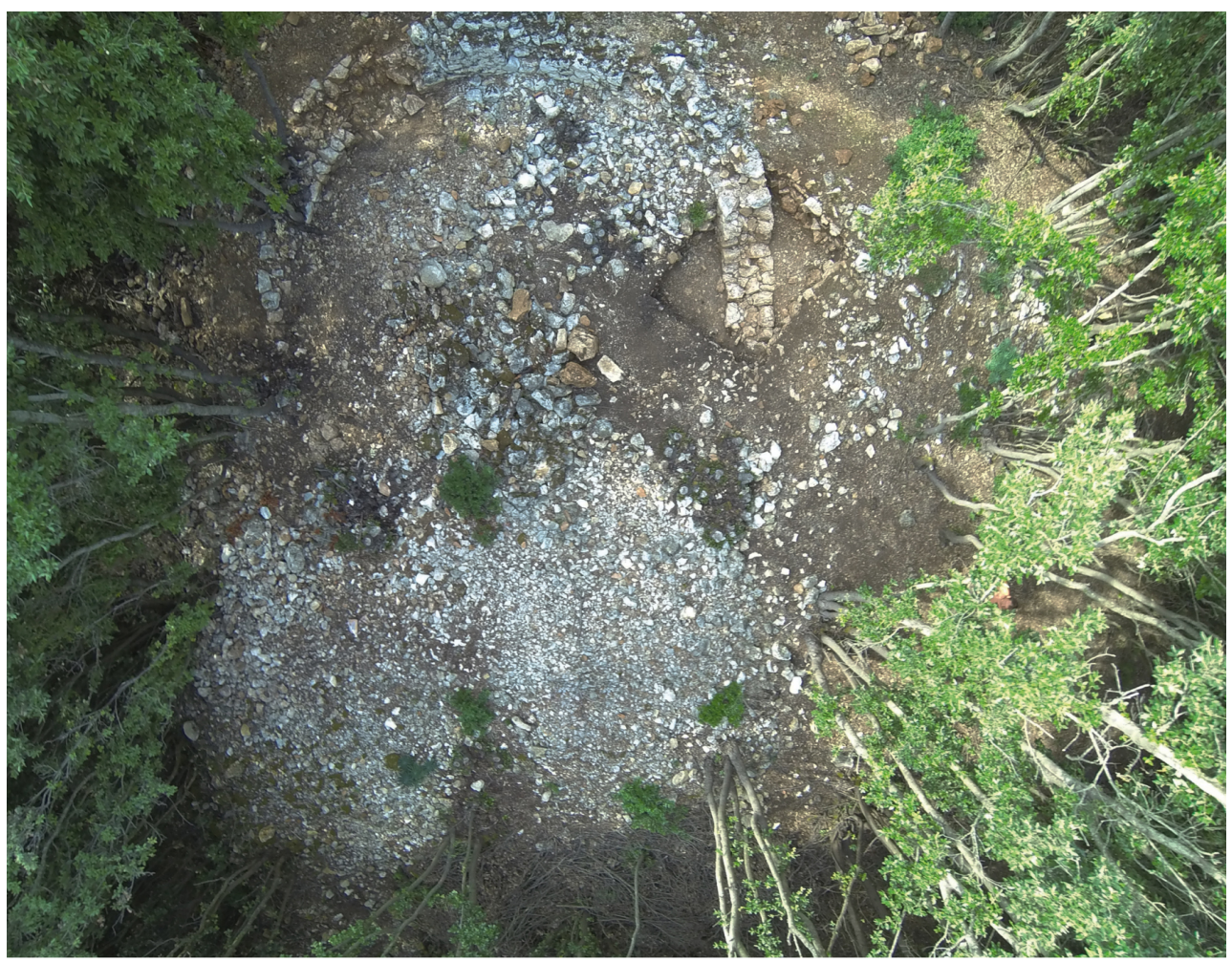

Cl. M. Vuković.

22 Les couches qui recouvraient ici les arases des maçonneries étaient assez simples et appartenaient toutes à la démolition du bâtiment. La première couche (7.1001) reconnue à l'extérieur du mur courbe 7.100 contenait un grand nombre de fragments de tegulae et de TCA, mais également des tessons de céramique identique à celles trouvées dans les couches inférieures du bâtiment $\mathrm{L}$ (notamment de la céramique noire avec des inclusions de quartz ou de calcaire), des coquillages et des ossements de faune. À l'intérieur du cercle formé par le mur 7.100, la couche 7.1002 était identique à la précédente, mais elle contenait aussi quelques spicae d'un sol en opus spicatum, ainsi qu'un fragment d'amphore de type Late Roman (IV $-\mathrm{VIII}^{\mathrm{e}} \mathrm{s}$.).

23 Le mur 7.100 suit un tracé curviligne de $\pm 5,90 \mathrm{~m}$ hors-oeuvre de diamètre. Le sondage n'a pas démontré l'existence d'un épaulement et on ignore par conséquent s'il s'agit d'une structure semi-circulaire outrepassée ou circulaire. La maçonnerie, en opus incertum, mesure 0,64 m de largeur; elle est conservée sur une élévation reconnue maximale de $0,68 \mathrm{~m}$.

Dans l'état actuel des données, il est envisageable que nous soyons en présence d'une construction de l'Antiquité tardive d'après le mode de construction et la présence d'une amphore de type Late roman. En revanche, le plan incomplet ne permet pas de déterminer s'il s'agit de l'abside outrepassée d'un édifice de culte - qui serait alors orienté au nord... -, de la tour d'angle d'une enceinte - mais dont l'épaisseur serait peu importante -, d'un bassin - aucune trace d'enduit hydraulique n'a été observée -, ou d'une tout autre construction de plan centré. 


\section{Étude et interprétation des élévations de Martinšćica 1 et 1 bis}

(fig. 7)

L'étude des élévations de la grande église de Martinšćica 1 et de son annexe Martinšćica 1 bis a été engagée en 2012.

Fig. 7 - Martinšćica, étude des élévations et proposition de restitution de la grande église.

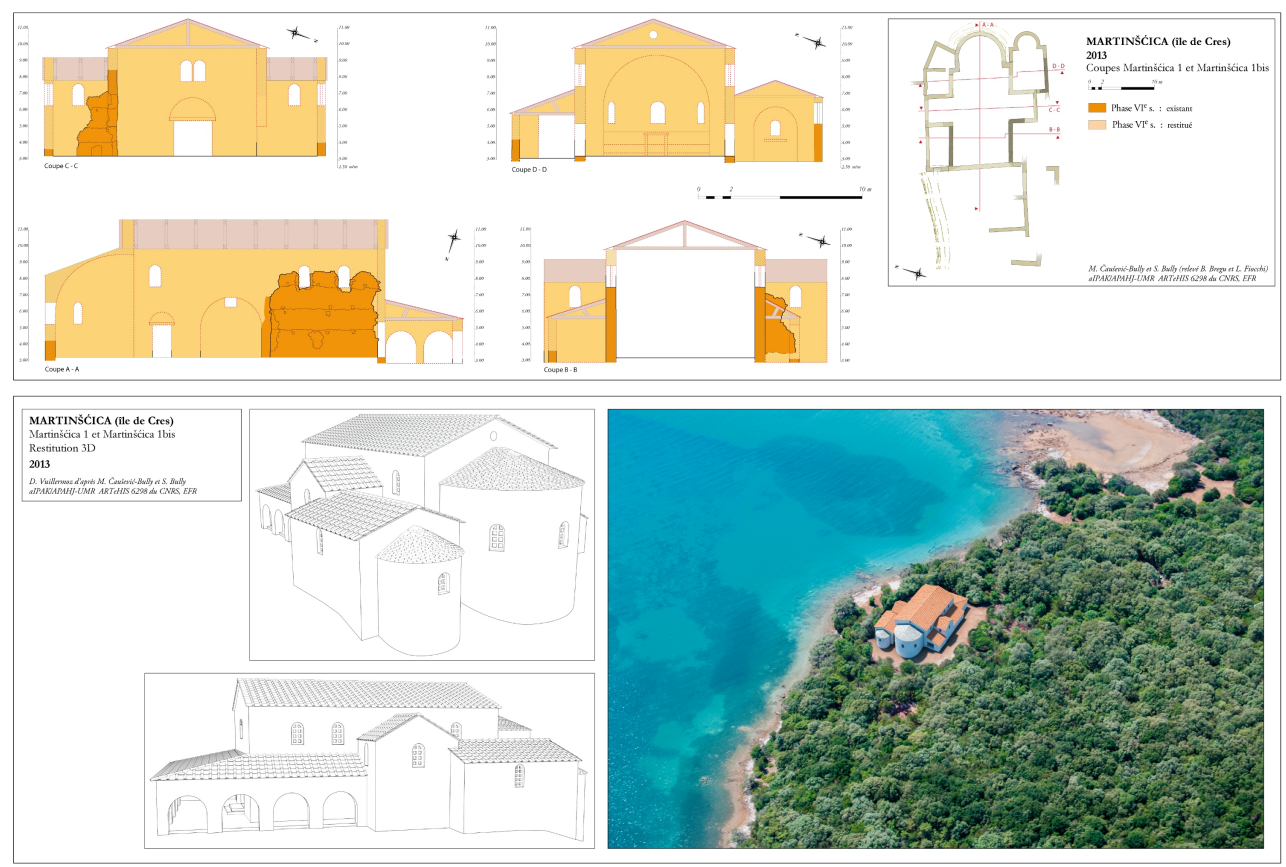

Restitution en 3D D. Vuillermoz, d'après M. Čaušević-Bully.

La documentation et les résultats obtenus en 2012 combinés aux plans de la grande église et de sa chapelle latérale sud élaborés en 2011, nous permettent de proposer une restitution des élévations des deux bâtiments cultuels par une série de coupe architecturales et une restitution en 3D.

\section{Volumes}

L'élévation de la grande église a pu être restituée principalement grâce aux murs gouttereaux de la nef dont on considère qu'ils sont préservés à environ $0,65 \mathrm{~m}$ en dessous de la corniche du toit d'après la position des baies. La nef unique de l'église ouvrait sur une grande abside que l'on situe à $0,85 \mathrm{~m}$ plus basse que les murs gouttereaux et le retour des épaulements. À l'articulation des deux volumes, nous proposons l'existence d'un grand arc triomphal. La hauteur des bras du transept devait être inférieure à celle des murs gouttereaux de la nef si l'on considère l'arrachement de l'arc d'entrée dans le bras sud. La chapelle Martinšćica 1bis possédait quand à elle tous les éléments nécessaires à la restitution de ses volumes. Seule la hauteur de l'abside a été projetée et restituée en fonction des volumes de la grande église. 


\section{Ouvertures}

28 de deux fenêtres. On restitue trois autres baies dans les bras du transept - une par côté -, tandis qu'une fenêtre de même taille et de même forme que celles des gouttereaux a dû exister de chaque côté des retours des épaulements. La restitution des ouvertures dans l'abside est entièrement hypothétique et repose sur des comparaisons avec des absides paléochrétiennes de la région (Sainte Marie d'Osor par exemple). Parmi les ouvertures proposées, seules les deux portes ouvertes dans les retours des épaulements et celle qui mène dans l'espace de la première annexe septentrionale sont archéologiquement prouvées. Les autres ouvertures sont restituées sur la base de parallèles relevées dans d'autres églises paléochrétiennes. Ainsi, nous proposons que la façade interne était percée d'un seul portail alors que le vestibule l'était par une arcature ouverte, permettant une libre circulation entre les différentes annexes.

La conjugaison des restitutions architecturales et des données archéologiques permet de proposer une datation de la grande église dans la première moitié du VI ${ }^{e}$ siècle. La construction de la chapelle latérale sud a dû intervenir peu après, dans le courant de la deuxième moitié $\mathrm{du} \mathrm{VI}{ }^{\mathrm{e}}$ siècle ou au $\mathrm{VII}^{\mathrm{e}}$ siècle au plus tard, si l'on considère le mode de construction et le choix d'une abside outrepassée. Les autres annexes, postérieures à la grande église, démontrent une longue période d'utilisation du complexe qu'il conviendra désormais de définir.

\section{Synthèse}

La stratigraphie rencontrée dans le sondage du bâtiment $\mathrm{L}$ indique que sa démolition s'est produite sans destruction violente, mais naturellement et progressivement en raison d'un manque d'entretien consécutif de son abandon. La chronologie relative des murs permet d'ores et déjà de distinguer trois phases de construction, témoignant d'une certaine durée d'occupation du site. Mais le mode de construction des maçonneries, avec des moellons équarris régulièrement assisés, contraste avec l' opus incertum qui caractérise les bâtiments de l'Antiquité tardive et du haut Moyen Âge, comme c'est le cas de l'église et de la construction de plan centrée, et plaide en faveur d'une datation du bâtiment $\mathrm{L}$ plus précoce, des $\mathrm{III}^{\mathrm{e}}-\mathrm{IV}^{\mathrm{e}} \mathrm{s}$. Les niveaux des sols, rehaussés par rapport aux niveaux des fondations et les reprises des maçonneries démontrent une longue durée d'utilisation du bâtiment, ce que confirme le mobilier monétaire et céramique des $\mathrm{IV}^{\mathrm{e}}-\mathrm{V}^{\mathrm{e}} \mathrm{s}$. Une phase d'occupation importante du site au $\mathrm{V}^{\mathrm{e}}$ siècle est désormais bien documentée par le résultat du sondage croisé à une datation obtenue par une analyse $\mathrm{C}^{14}$ réalisée sur une tombe maçonnée découverte en prospection sur la grève. La datation réalisée sur le collagène d'un os long donne une fourchette chronologique entre 407 et 542. La construction en opus incertum de Martinšćica 2 va également dans le sens d'une phase d'occupation de l'Antiquité tardive. En dépit de l'absence de mobilier, on ne doit pas cependant exclure une occupation postérieure à l'Antiquité tardive si l'on considère la présence de trous de piquet et de poteau perçant les sols de mortier du bâtiment $\mathrm{L}$.

31 Ces premiers résultats plaident en faveur d'une fonction funéraire et par conséquent religieuse du site antérieure au milieu $\mathrm{du} \mathrm{VI}^{\mathrm{e}} \mathrm{s}$. et à la reconquête justinienne des îles du Kvarner. Mais on ignore encore si cette occupation est contemporaine de la grande église de plan en croix grecque ou d'une phase antérieure. Il est en revanche désormais

Chronique des activités archéologiques de l'École française de Rome , Balkans 
fortement probable que le complexe de Martinšćica ait été à l'origine une villa maritime, avant d'être transformé en site ecclésial paléochrétien. Une fonction monastique est tout à fait envisageable, même si elle reste à démontrer sur la base, notamment, d'une occupation médiévale. Une monnaie du XIII ${ }^{\mathrm{e}}$ s. découverte sur le site en prospection est à ce jour le seul témoignage d'une occupation (?) tardive9. Outre la prise en compte de la localisation du complexe au fond d'une baie isolé, des dimensions et du parti architectural singulier de la grande église, rappelons que l'hypothèse d'un monastère existant avant 1046 est suggérée par une brève mention relevée dans la Vie de saint Gérard de Sagredo - abbé de Saint-Georges dans la lagune de Venise -, où il est fait état d'une escale, entre Venise et Zadar, dans un monastère placé sous la dédicace de l'apôtre des Gaules ${ }^{10}$. La fouille du bâtiment de plan centré - Martinšćica 2 - pourrait être déterminante pour l'interprétation du site.

\section{Saint-Jean-Saint-Pantaléon, îlot d'Oruda (première campagne) : état des lieux}

\section{Présentation}

Le site d'Oruda a été intégré dans le programme de recherche sur le «Monachisme insulaire dans l'archipel du Kvarner » dès son élaboration en 2010. Nous avions alors donné une courte description du site après une première prospection et un rapide inventaire des données bibliographiques - assez limitées par ailleurs ${ }^{11}$. Ainsi, l'îlot d'Oruda, au large de Mali Lošinj, se situe à un peu plus de 600 mètres de l'îlot de Palacol sur lequel se trouvent des vestiges d'une grande construction de plan quadrangulaire interprétée comme un fortin byzantin. Oruda mesure environ $1100 \mathrm{~m}$ de longueur par $420 \mathrm{~m}$ de largeur, soit une surface de plus de $46 \mathrm{ha}$, et culmine à seulement $13 \mathrm{~m}$ audessus du niveau de la mer (fig. 8). 
Fig. 8 - Oruda, vue générale de l'île (extrait d'image ARKOD) et localisation du site.

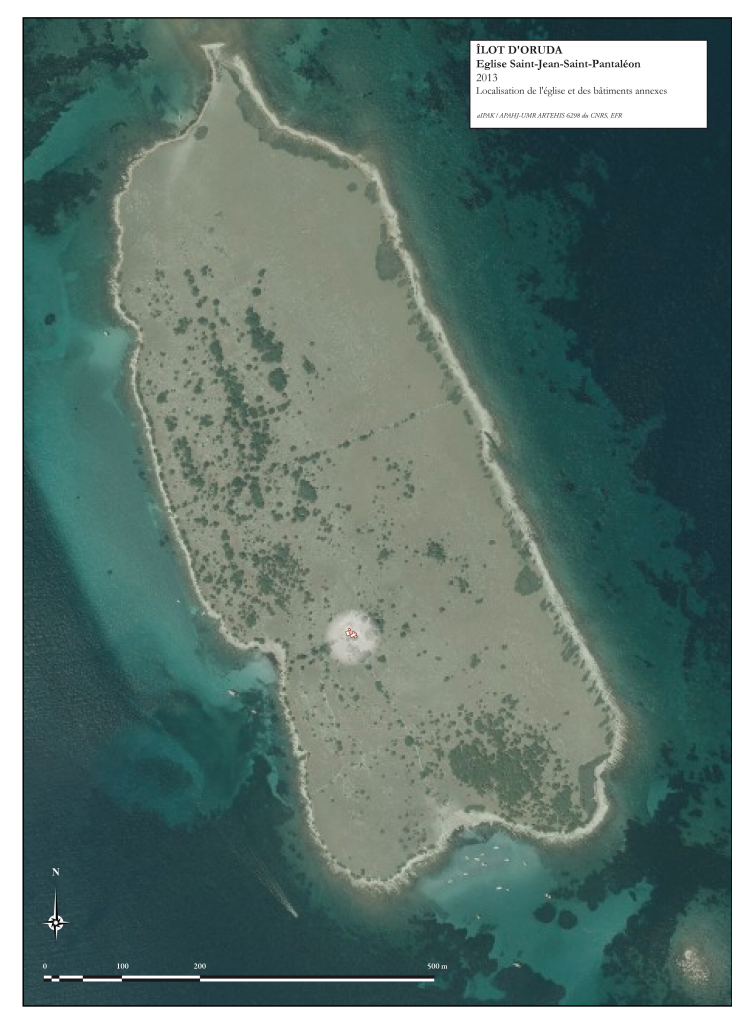

Son accès est rendu difficile par une grève rocheuse continue interrompue par quelques petites criques vaseuses. L'̂̂lot inhabité se présente comme un vaste champ de chardons et de buissons épineux ponctué de quelques bosquets. Au centre, les ruines d'une église sont dissimulées et envahies par une importante végétation. Il s'agit d'un édifice de petites dimensions, dont l'abside centrale semi-circulaire est partiellement conservée en élévation alors que le reste de l'église, dont une absidiole au sud, est enfoui sous un monticule de pierres. Un plan partiel et une esquisse de restitution axonométrique ont été publiés par A. Mohorovičić en $1957^{12}$. À partir de l'observation des vestiges, Mohorovičić suggérait un plan d'église à trois nefs. Toujours selon les recherches de ce dernier, le vocable primitif de l'église aurait été Saint-Pantaléon, référence directe à l'église d'Orient ${ }^{13}$ et à un ermitage de quelques moines sur le petit îlot.

C'est sur la base de ces éléments que nous avons engagé la campagne 2013 ; celle-ci s'est déroulée en trois temps. Avant tout, plusieurs jours ont été consacrés à un important travail de débroussaillage du site - et plus particulièrement de l'église - avant d'ouvrir des sondages localisés puis de relever les élévations grâce à un scanner laser ${ }^{14}$ (fig. 9). 
Fig. 9 - Oruda, vue générale des vestiges de l'église après débroussaillage.

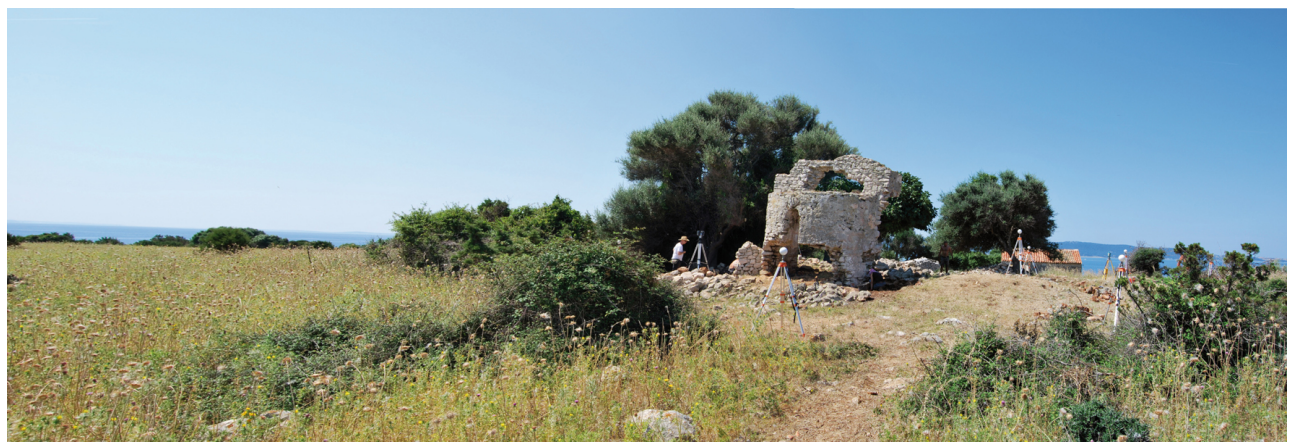

Cl. M. Čaušević-Bully.

L'objectif de l'opération était d'établir une nouvelle documentation archéologique de l'église afin d'en proposer un plan. Il s'agissait également d'évaluer son environnement immédiat afin de définir s'il s'agissait d'un édifice isolé ou accompagné d'autres constructions.

\section{Principaux résultats}

\section{Abside (mur 101) (fig. 10 et 11)}

L'abside de l'église mesure $2,80 \mathrm{~m}$ de largeur au niveau de sa corde pour une profondeur de $1,90 \mathrm{~m}$ et une épaisseur de $0,46 \mathrm{~m}$.

Fig. 10 - Oruda, relevé de l'abside de l'église par scanner 3D.

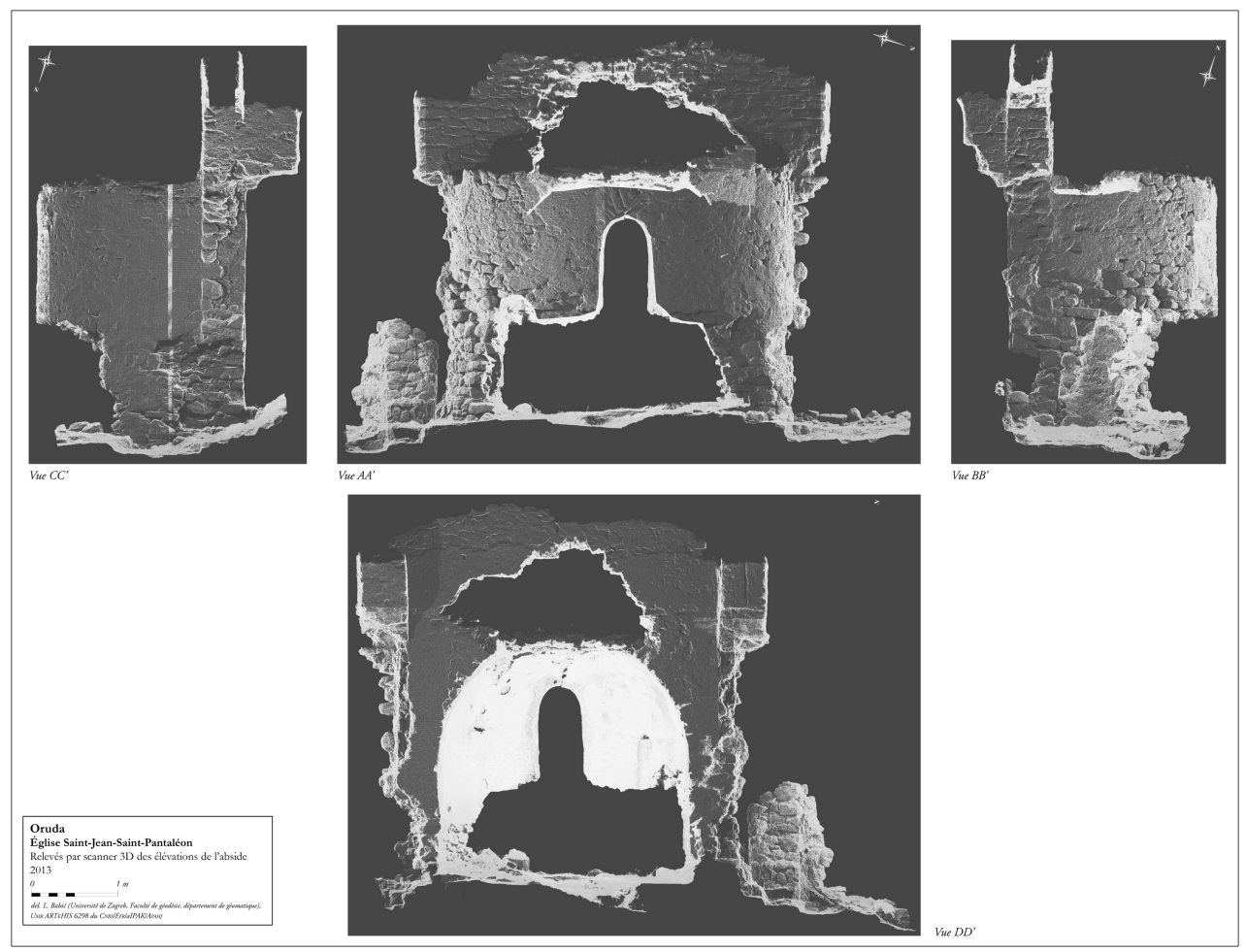

L. Babić, Université de Zagreb, Faculté de géodésie, département de géomatique. 
Fig. 11 - Oruda, plan masse de l'église et relevé pierre à pierre.

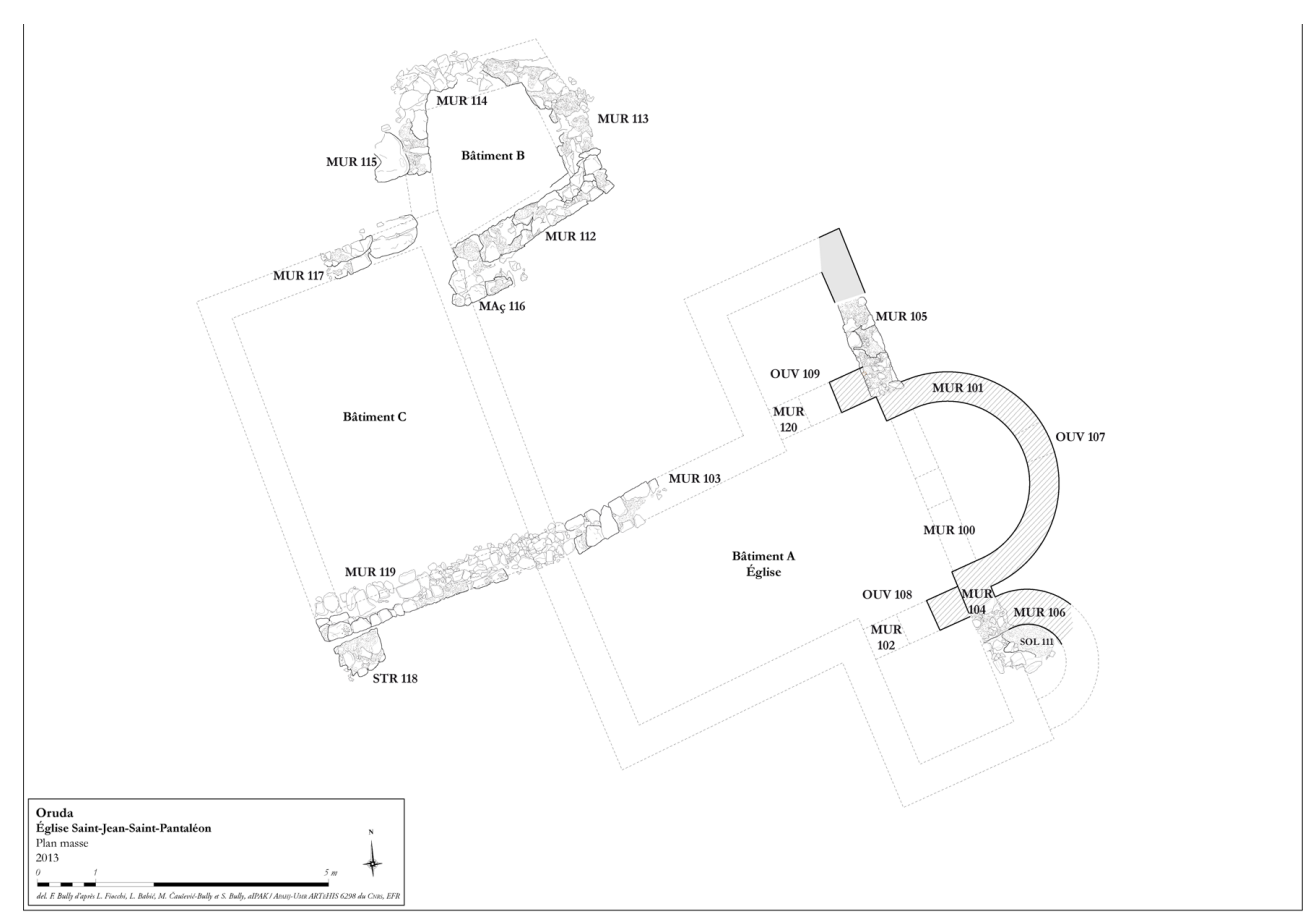

Infographie F. Bully, relevés L. Fiocchi, d'après M. Čaušević-Bully et S. Bully.

Elle est construite en moellons de taille irrégulière grossièrement équarris et se caractérise par l'utilisation sporadique de briques et de tegulae - surtout présents dans la partie haute de la maçonnerie. Le parement externe est enduit d'un mortier assez grossier marqué par des joints tirés au fer horizontaux et verticaux simulant un appareil plus ou moins régulier ; l'épaisseur moyenne du trait est de $6 \mathrm{~mm}$, tandis que l'espacement entre les joints horizontaux forme de fausses assises de 24 à $29 \mathrm{~cm}$ en partie haute pour $\pm 18 \mathrm{~cm}$ en partie basse. À l'intérieur, un fin enduit chaulé laisse la place à un enduit plus grossier à la jonction du mur de l'abside avec sa voûte en cul-defour. La voûte elle-même est construite en moellons calcaires, en briques et en tegulae remployées. Dans l'arc diaphragme (ARC 110) alternent des briques rouge pâle et jaune - L. $28 \mathrm{~cm}$, ép. $7 \mathrm{~cm}$ - et des moellons de taille moyenne selon un rythme d'environ un moellon pour quatre briques. Deux impostes (10000 - imposte nord, et 10001 - imposte sud) situées à $0,85 \mathrm{~cm}$ sous les départs de l'arc de l'abside, semblent avoir été insérées dans une phase postérieure d'après une reprise de maçonnerie (10002) observable sous l'imposte nord et un changement de liant mortier.

L'abside est percée d'une fenêtre axiale en plein cintre, légèrement brisée à l'extérieur, de médiocre facture constructive (OUV 107, 0,59 x 1,08 m). Son arc et ses montants sont formés de briques et de tuiles en remploi, ainsi que de moellons rectangulaires non taillés de forme irrégulière, allongés ou grossièrement rectangulaires. La baie accueillait un transenne formé de deux plaques d'après les négatifs inscrits dans l'enduit bien préservé des tableaux. La partie inférieure possédait une base d'environ $13 \mathrm{~cm}$ de largeur par 9,5 $\mathrm{cm}$ de hauteur supportant une partie centrale de $0,68 \mathrm{~m}$ de hauteur par $4 \mathrm{~cm}$ de largeur. La claustra rectangulaire devait être surmontée par une seconde plaque en forme de lunette dans l'arc de la baie. 


\section{Annexes latérales ou transept} localisé par un sondage permet de restituer une petite nef d'environ 3,10 $\mathrm{m}$ de largeur. Le mur 103 est très mal préservé et incertain par endroits ; seul un segment de 1,80 m de longueur par $0,58 \mathrm{~m}$ de largeur a été reconnu dans le prolongement des murs diaphragmes des espaces latéraux, bien que légèrement décalé d'une vingtaine de $\mathrm{cm}$ à l'intérieur. Le segment de maçonnerie se poursuit en direction de l'ouest, mais en s'inclinant légèrement en direction $\mathrm{du}$ nord. Nous interprétons ce changement d'orientation comme l'emplacement présumé de la façade. La nef ainsi restituée mesurerait environ 4,20 m de longueur jusqu'aux « annexes latérales».

\section{Constructions annexes (bâtiment B et C)}

43

Comme nous venons de l'évoquer, le potentiel mur gouttereau nord 103 se prolonge à l'ouest à travers une maçonnerie (119) reconnue sur une longueur de 4,64 m par 0,47 m de largeur avant de s'interrompre sur un angle droit au nord. Son parement sud est conservé sur seulement deux assises alors qu'un seul moellon permet de restituer le parement nord et ainsi de connaître la largeur du mur. L'angle sud-ouest présumé est conservé sur quatre assises ou l'on distingue nettement des traces de joints tirés au fer dans l'enduit, semblables à ceux observés sur le parement extérieur de l'abside. Une structure maçonnée de 0,83 x 0,61 m (118), préservée sur trois assises, est adossée contre l'angle du mur 119. La fonction de cette structure demeure indéterminée. 

grand nombre de questions demeurent en suspens. Sur la base des vestiges observés en élévation et reconnus en sondage, nous proposons de ne pas retenir l'hypothèse émise par A. Mohorovičić d'une basilique à trois nefs, mais plutôt celle d'un petit édifice de culte formé d'une courte nef unique dotée de deux espaces latéraux et ouvrant sur une abside semi-circulaire. Les dimensions externes restituées sont de $9 \mathrm{~m}$ de longueur estouest par 9,50 m nord-sud au niveau des espaces latéraux pour une nef de 4,25 $\mathrm{m}$ de large. Le plan que nous présentons doit cependant être considéré comme une nouvelle étape dans la documentation du site et une hypothèse de travail engageant à une poursuite des investigations (fig. 12). 
Fig. 12 - Oruda, proposition de restitution du plan de l'église et localisation des vestiges attestés ou supposés.

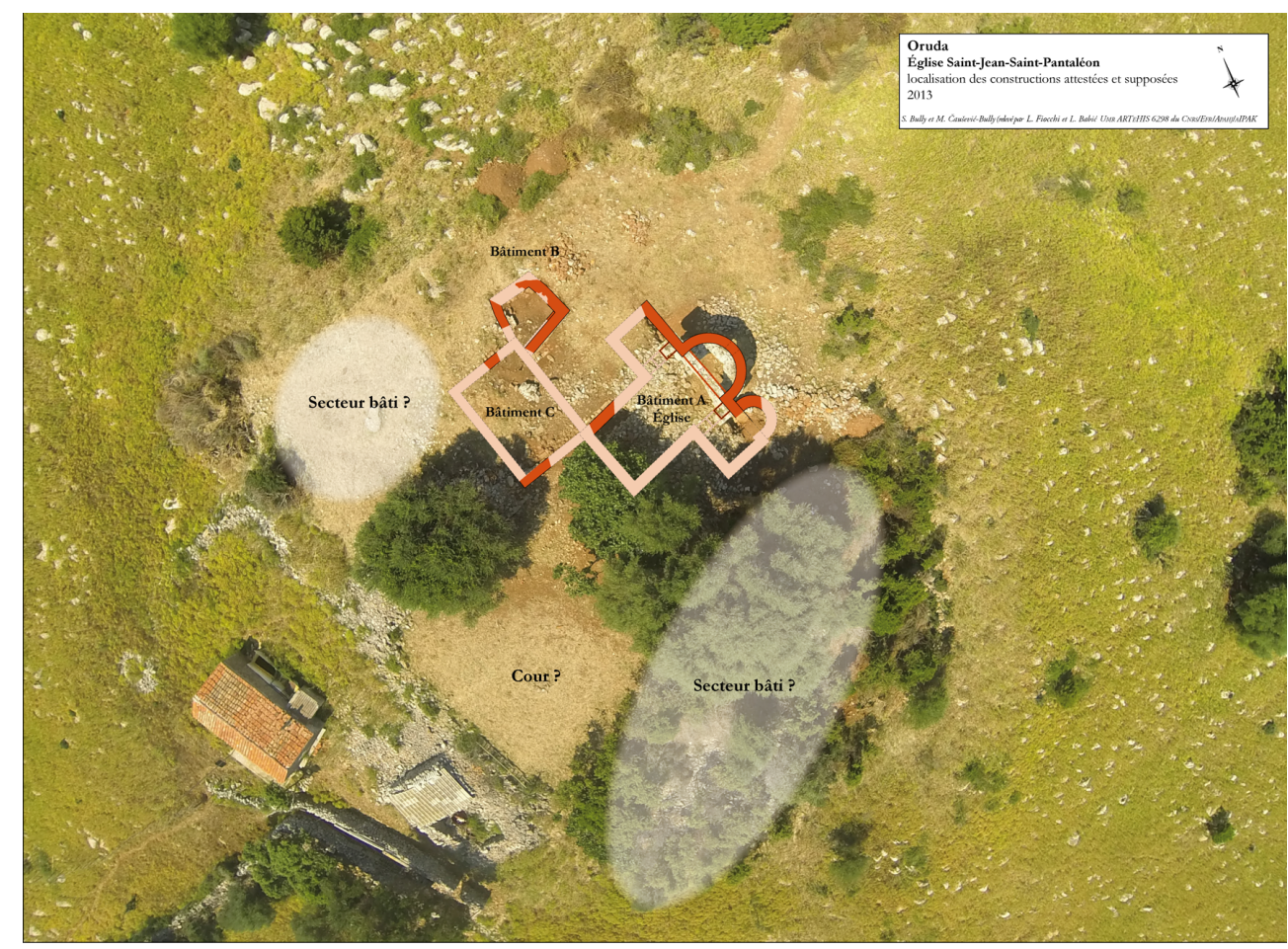

Infographie et plan L. Fiocchi, d'après M. Čaušević-Bully et S. Bully.

Pour une première phase, Saint-Jean d'Oruda s'inscrirait parfaitement en typologie dans la famille de ces simples chapelles à nef unique et à abside semi-circulaire que l'on identifie à des petits sanctuaires paléomonastiques. Elle s'en distingue pourtant nettement par les aménagements latéraux entre l'abside et la nef. Leur présence justifie la situation des deux baies hautes en partie conservées qui ouvraient au-dessus de leur toiture. Mais l'arrachement des maçonneries ne permet pas de déterminer s'il s'agit d'annexes latérales fermées sur l'espace de la nef - à l'exception d'une porte - ou de bras d'un transept ouverts par un arc. Mais quoi qu'il soit, nous sommes en présence d'un plan assurément atypique pour la région, qui nous renvoie plutôt à des modèles connus en France, en Suisse, ou encore en Orient, pour la période de l'Antiquité tardive. Les indices recueillis comme l'analyse de la construction ne permettent cependant pas de préciser la datation de l'église entre l'Antiquité tardive et le haut Moyen Âge. Il est en revanche assuré que le site a connu une phase d'occupation durant l'Antiquité tardive, comme l'indique la découverte de céramique de type Late Antique Roman. Les très nombreuses tegulae et briques qui jonchent le sol ou qui sont réutilisées dans la construction de l'église nous interpellent également sur une occupation durant l'Antiquité ou/et l'Antiquité tardive. Il serait hasardeux d'aller au-delà de ce constat sans une étude archéologique plus poussée et dans l'attente de la datation $\mathrm{C}^{14}$ engagée sur un charbon de bois pris dans le mortier de la construction.

En ce qui concerne la fonction du site, il est fortement probable que l'église ait pris place au sein d'autres constructions localisées sur son flanc nord. La micro-topographie du site laisse également supposer l'existence de construction à l'ouest du bâtiment $C$ (dépressions et talus) ainsi qu'au sud de l'église (tas d'épierrement dans la végétation). Pour l'église, l'adjonction d'une absidiole latérale au sud indique une évolution dans les 
besoins du culte par une amplification du chevet et une utilisation sur une longue durée. Ces nouvelles données vont donc dans le sens de l'hypothèse d'un petit établissement monastique, mais sans la démontrer de manière assurée.

\section{Vestiges sur l'îlot de Lukovac, baie de Crnika, île de Rab (première campagne) : état des lieux}

\section{Présentation}

L'îlot de Lukovac est situé dans la baie de Crnika, sur la façade orientale de l'île de Rab. Il mesure moins d'une centaine de mètres de longueur par une soixantaine de mètres de largeur au maximum. Cerné de falaises dans sa quasi-totalité, le petit îlot domine la baie d'une vingtaine de mètres de hauteur; on accède au sommet par son flanc nord grâce à un court sentier fortement pentu. La végétation est essentiellement constituée d'herbes hautes parsemées de buissons épineux et de ronces; des roseaux poussent sur quelques mètres carrés à l'extrémité est de l'îlot. Le toponyme de « Lukovac » pourrait dériver du mot croate "luka», qui signifie "port», ou encore «luk» qui signifie «oignon $»^{15}$. Mais le nom n'est pas sans rappeler le vocable de Luc et on distingue au centre de l'îlot une petite abside presque entièrement dissimulée par la végétation (fig. 13).

Fig. 13 - Lukovac, vue générale de lîle et localisation du site (extrait d'image ARKOD).

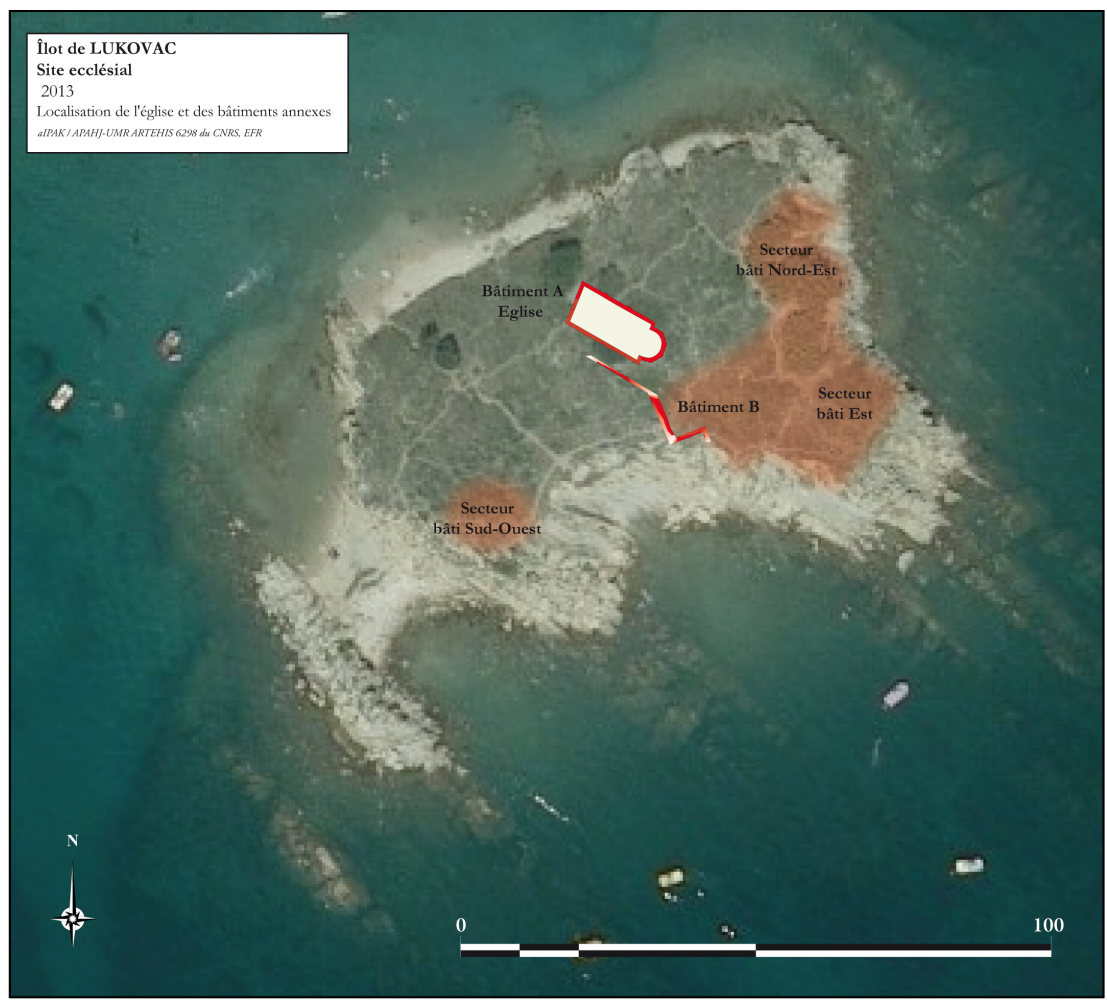

Plan L. Fiocchi, d'après M. Čaušević-Bully et S. Bully.

51 Les vestiges de cet édifice sont très mal connus et ne figurent sur aucun plan ; ils sont néanmoins répertoriés dans l'inventaire des sites archéologiques du Service régional de la conservation du patrimoine culturel de Rijek ${ }^{16}$. Lors de notre première visite du site 
en 2010 nous avions supposé l'existence d'une abside de plan polygonal à l'extérieur, peut-être dotée de lésènes. Outre la petite église, nous avions noté des vestiges de maçonneries en rebords de falaises semblant former comme un mur de clôture ou d'enceinte et des murs de terrasses (?) sur le flanc nord de l'îlot. Sa partie orientale est marquée par plusieurs dépressions, de plans grossièrement quadrangulaires, qui pourraient correspondre à des constructions. Le "bosquet» de roseaux dessine également un plan quadrangulaire régulier qui pouvait indiquer l'emplacement d'une citerne.

Pour cette opération, nous avons été contactés par la commune de Lopar, propriétaire de l'îlot, qui a émis le souhait de favoriser notre démarche. Nous avons ainsi bénéficié d'une aide logistique pour le transport de l'équipe et des outils sur l'île, ainsi que pour le nettoyage superficiel de la végétation ${ }^{17}$. L'objectif de cette première et courte intervention était de dresser un premier plan de l'église et d'établir un état des lieux du site - qu'il conviendra de compléter et de terminer. En dissimulant les arases de murs, la végétation a en effet fortement limité les relevés au tachéomètre laser dans le temps imparti à cette opération.

\section{Résultats}

Après le nettoyage superficiel des ronces et des hautes herbes qui recouvraient la totalité des maçonneries, nous avons pu dégager grossièrement le plan de l'église et mieux observer les vestiges de l'architecture environnante.

L'église se présente comme un édifice à nef unique - dim. ext. $\pm 13,50 \times 7,50 \mathrm{~m}-$, dotée d'une façade en biais par rapport aux murs gouttereaux, ouvrant sur une abside non pas polygonale mais semi-circulaire légèrement saillante - diam. à la corde $4,50 \mathrm{~m}$, prof. 3,60 m (fig. 14).

Fig. 14 - Lukovac, plan masse de l'église.

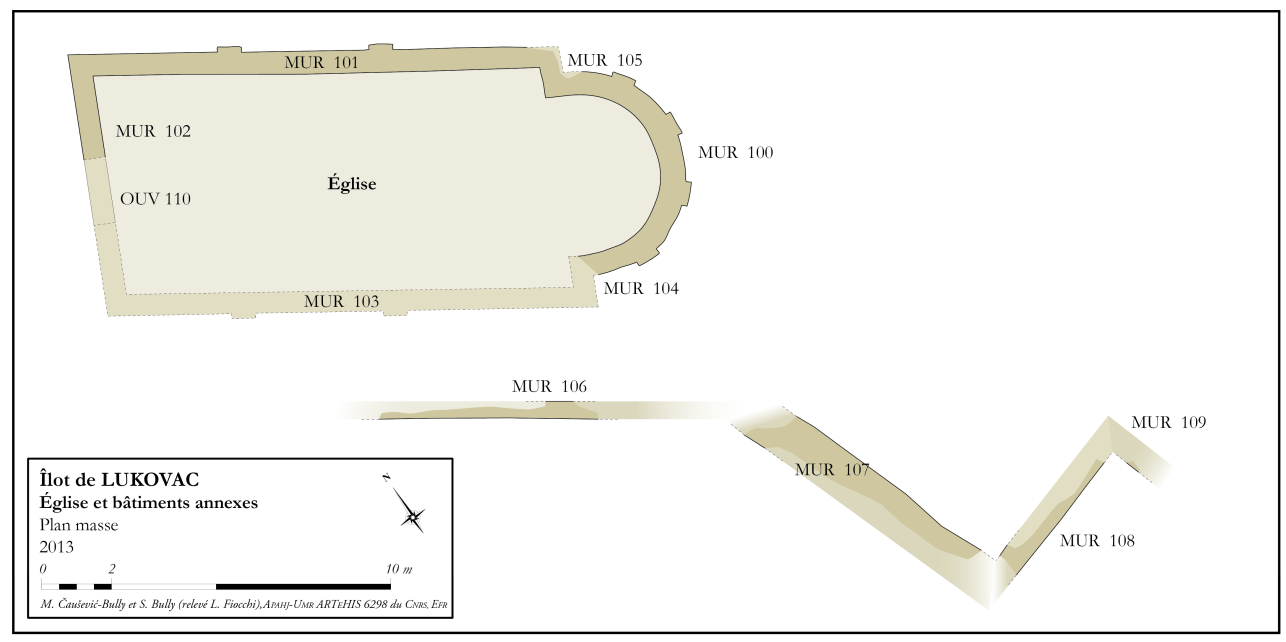

Infographie et plan L. Fiocchi, d'après M. Čaušević-Bully et S. Bully.

L'extrados de l'abside est rythmé par quatre lésènes, alors que les murs gouttereaux en possèdent deux chacun sur leurs parements externes. Une seule porte en façade a été reconnue. Les maçonneries en opus incertum mesurent $0,64 \mathrm{~m}$ de largeur pour la nef et $0,72 \mathrm{~m}$ pour l'abside. La largeur des murs et la présence de lésènes nous conduisent à 
restituer une voûte en cul-de-four dans l'abside. On ignore la hauteur conservée des élévations.

$\mathrm{Au}$ chevet et au nord de l'église se développe un petit complexe de bâtiments annexes, organisés autour de l'église et respectant la configuration du terrain. Nous avons reconnu en prospection plusieurs murs dans la partie septentrionale de l'île, ainsi qu'un épais sol de mortier, visible en coupe du côté nord de l'îlot. Le sol en question, recouvert d'une couche de terre noire très organique, est couvert par le bosquet de roseaux qui poussent là en raison de l'humidité préservée par le revêtement de mortier et la terre fertile. Le mobilier recueillit dans ce secteur date assurément de l'Antiquité tardive; c'est le cas notamment d'une céramique peignée qui rappelle les formes découvertes à Martinšćica, à Oruda, ou encore à Mirine ${ }^{18}$. On discerne d'ores et déjà au moins trois différents secteurs sur l'îlot qui accueillaient des constructions : un premier au chevet de l'église, un second dans son prolongement en direction du nord-est, et un troisième sous les roseaux. Des maçonneries sont également reconnues sur le flanc sud de l'église : un mur parallèle au gouttereau sud a dû créer une sorte de portique ou de clôture, tandis qu'un petit segment de mur à l'extrémité méridionale de l'île laisse supposer que la totalité de la surface de Lukovac était lotie.

\section{Synthèse}

57 Le plan de l'église, avec ses lésènes, et son mode de construction en opus incertum évoque une datation dans la deuxième moitié $\mathrm{du} \mathrm{VI}^{\mathrm{e}} \mathrm{s}$. Les tessons de céramique recueillis dans le secteur nord, dans la couche recouvrant directement les sols en mortier, sont datables entre le $\mathrm{IV}^{\mathrm{e}}$ et le $\mathrm{VII}{ }^{\mathrm{e}} \mathrm{s}$. Le site nécessiterait un examen plus approfondi afin de confirmer cette première proposition de datation, mais les données recueillies à ce jour plaident donc en faveur d'une occupation de l'îlot durant l'Antiquité tardive.

Cette première campagne de prospection ne nous a pas permis de travailler d'avantage sur la fonction du petit complexe. La configuration générale du site conviendrait cependant à un fortin byzantin contrôlant l'accès à la baie de Crnika ou à un monastère. Le contexte dans lequel s'inscrit l'îlot rappelle globalement les sites fortifiés liés au limes maritime byzantin qui est créé à partir de la fin des guerres gothiques. Et il convient de signaler l'existence, dans cette même baie, du promontoire de Kaštelina situé dans la proximité immédiate de Lukovac, et dont le toponyme est souvent associé à un site fortifié. Cependant, on ne peut pas exclure qu'à l'origine ce toponyme soit celui du Lukovac actuel, avant un glissement sur l'îlot voisin. Mais Lukovac ne partage pas cette caractéristique commune aux autres sites qui occupent des positions perchées permettant une surveillance des routes maritimes. Aussi, l'hypothèse d'un petit monastère n'est pas à exclure, justement en raison de sa situation, comme l'affirme aussi Petar Skok ${ }^{19}$. Un sondage archéologique au chevet serait nécessaire afin de s'assurer de la présence ou de l'absence d'inhumations, ce qui pourrait apporter un élément de réponse à la question de la nature de l'occupation. 


\section{Fulfinum - Mirine (Omišalj) baie de Sepen, île de Krk (seconde campagne) : fouille d'un mausolée}

\section{Présentation}

Alors que toutes les villes antiques insulaires de la région ont connu un développement continu jusqu'à nos jours, la ville de Fulfinum, dans la baie de Sepen, création ex nihilo du I ${ }^{\text {er }} \mathrm{s}$ ap. J.-C., paraît avoir été abandonnée dès la fin de l'Antiquité. Les installations de l'Antiquité tardive et du Moyen Âge présentes dans la baie ne semblent pas devoir être mises en lien avec l'ancien espace urbain antique. C'est notamment le cas d'un monastère bénédictin connu par les textes à partir du XIII ${ }^{\mathrm{e}}$ s., placé sous la titulature de Saint-Nicolas, mais dont on pressent une fondation plus ancienne - au moins du XI ${ }^{\mathrm{e}} \mathrm{s}$. qui aurait pris place dans l'important complexe paléochrétien de Mirine. Cet ensemble est formé par une grande basilique du $\mathrm{V}^{\mathrm{e}} \mathrm{s}$. bordée d'une cour à portiques des $\mathrm{VI}^{\mathrm{e}}-\mathrm{VII}^{\mathrm{e}} \mathrm{s}$. sur son flanc sud (fig. 15).

Fig. 15 - Mirine-Fulfinum, plan topographique du site.

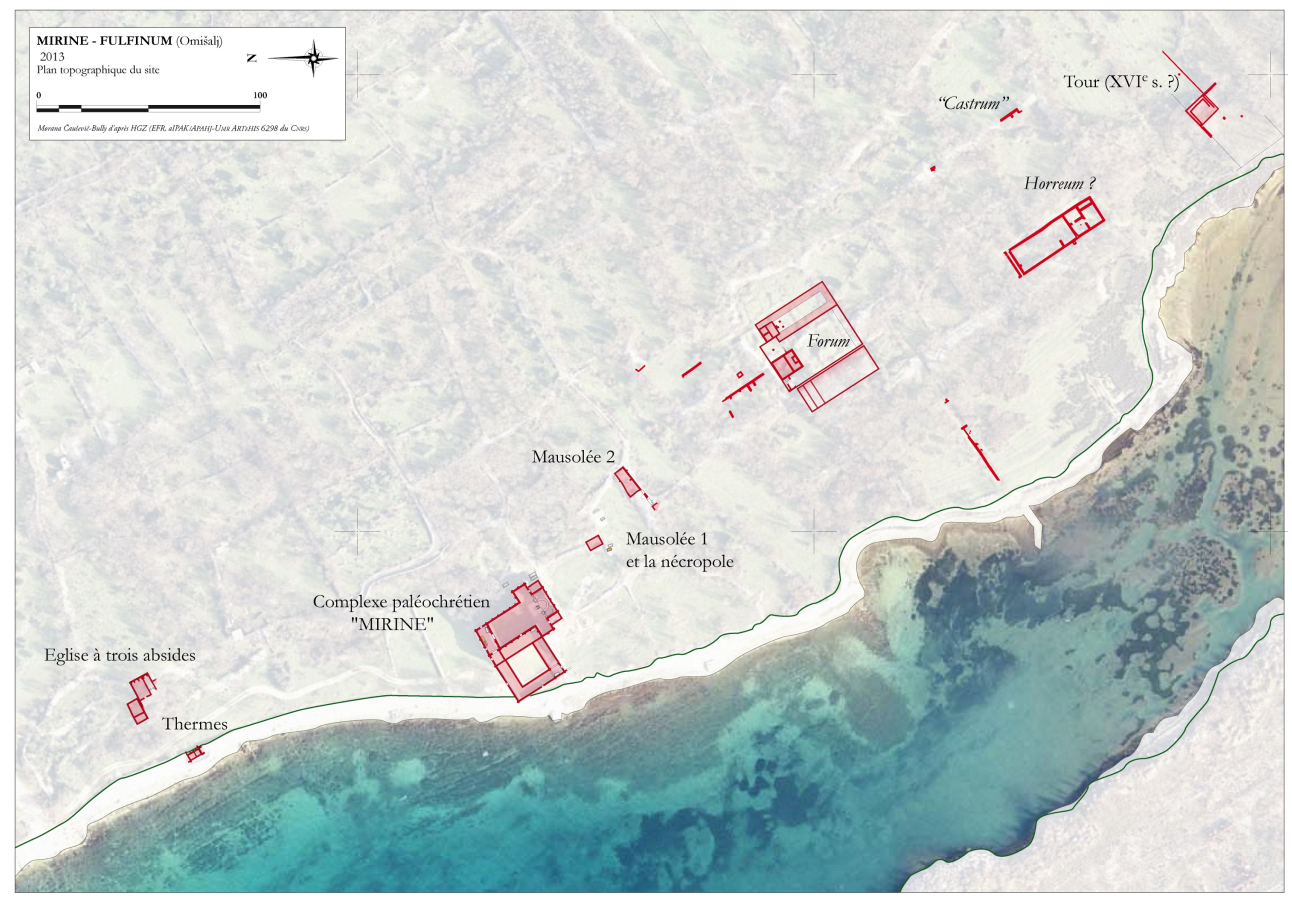

Plan M. Čaušević-Bully.

Un second complexe à environ $150 \mathrm{~m}$ à l'ouest est formé par une église des $\mathrm{IX}^{\mathrm{e}}-\mathrm{XI}^{\mathrm{e}} \mathrm{s}$. aménagée dans une construction de l'Antiquité tardive. Les prospections géophysiques engagées en 2012 ont révélé la présence d'une probable citerne dans l'atrium paléochrétien - lui accordant une fonction d'accueil? - et d'une possible villa suburbaine à l'emplacement de l'église du haut Moyen Âge.

61 La campagne de cette année a porté sur la fouille d'un bâtiment situé entre le forum de Fulfinum et l'église paléochrétienne de Mirine. Le bâtiment, enfoui sous un tas d'épierrement, avait été repéré depuis plusieurs années et interprété comme une 
potentielle construction en lien avec le monastère en raison de sa situation et de son ampleur présumée.

62 Le tas d'épierrement recouvrant la construction a été enlevé mécaniquement durant l'automne 2012 sous surveillance archéologique. La fouille entreprise au mois d'avril 2013 avait pour objectif de définir le plan de la construction et sa fonction. Seule la moitié ouest du bâtiment a été fouillée cette année, mais les données recueillies indiquent d'ores et déjà qu'il s'agit en fait d'un mausolée (fig. 16).

Fig. 16 - Mirine-Fulfinum, vue du mausolée depuis le sud-ouest après les travaux.

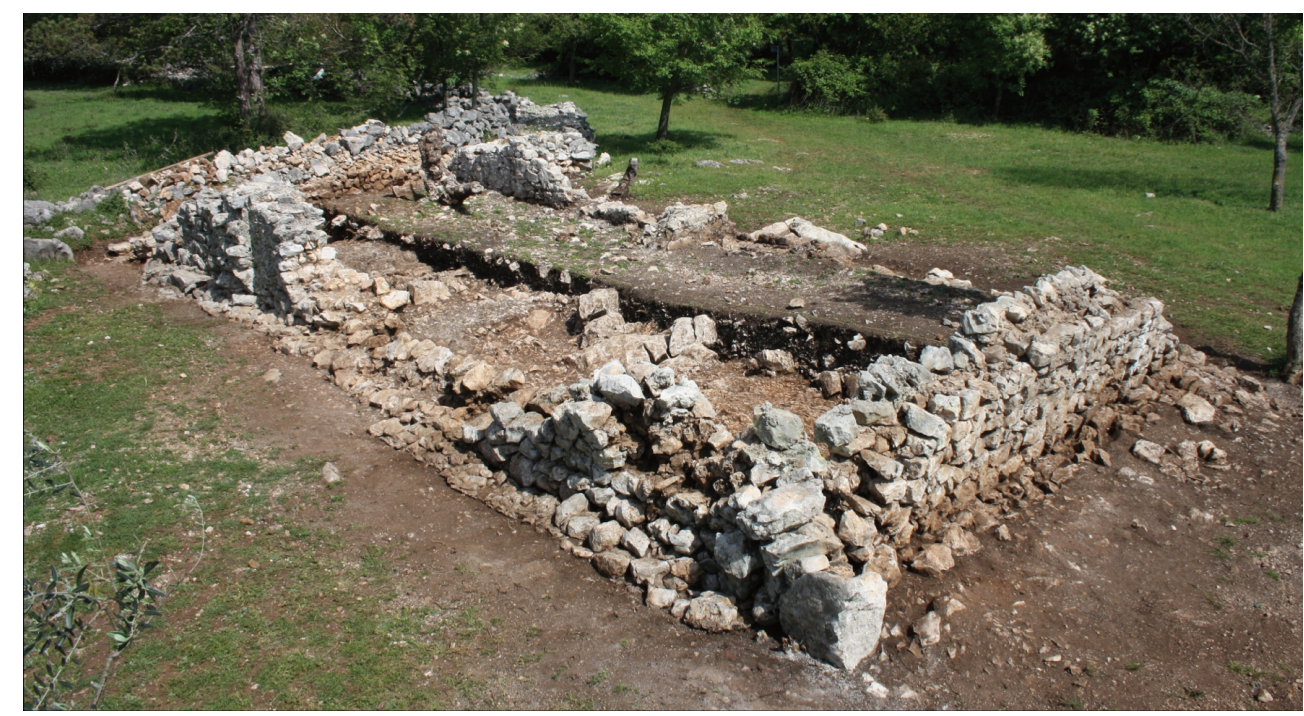

Cl. A. Mougin.

63 Celui-ci prend place au sein d'une nécropole de l'Antiquité tardive qui se développe à l'est de la basilique paléochrétienne. Cette aire d'inhumation est connue depuis des recherches effectuées par une équipe de l'UNESCO et du Centre de Préservation des Monuments de Rijeka, sous la direction de Claudia Pizzinato et de Nino Novak, dans les années 1990. Les recherches archéologiques avait alors a livré une série de tombes familiales, en amphore, maçonnées, ou en pleine terre, ainsi qu'un petit bâtiment de plan rectangulaire, sans tombe dans le sol, interprété comme une memoria.

\section{Principaux résultats (fig. 17)}

Dans une première phase, le mausolée (Bâtiment II.1A) était de plan rectangulaire de 4,6 x 6 m hors-œuvre. 
Fig. 17 - Mirine-Fulfinum, proposition de phasage du mausolée.
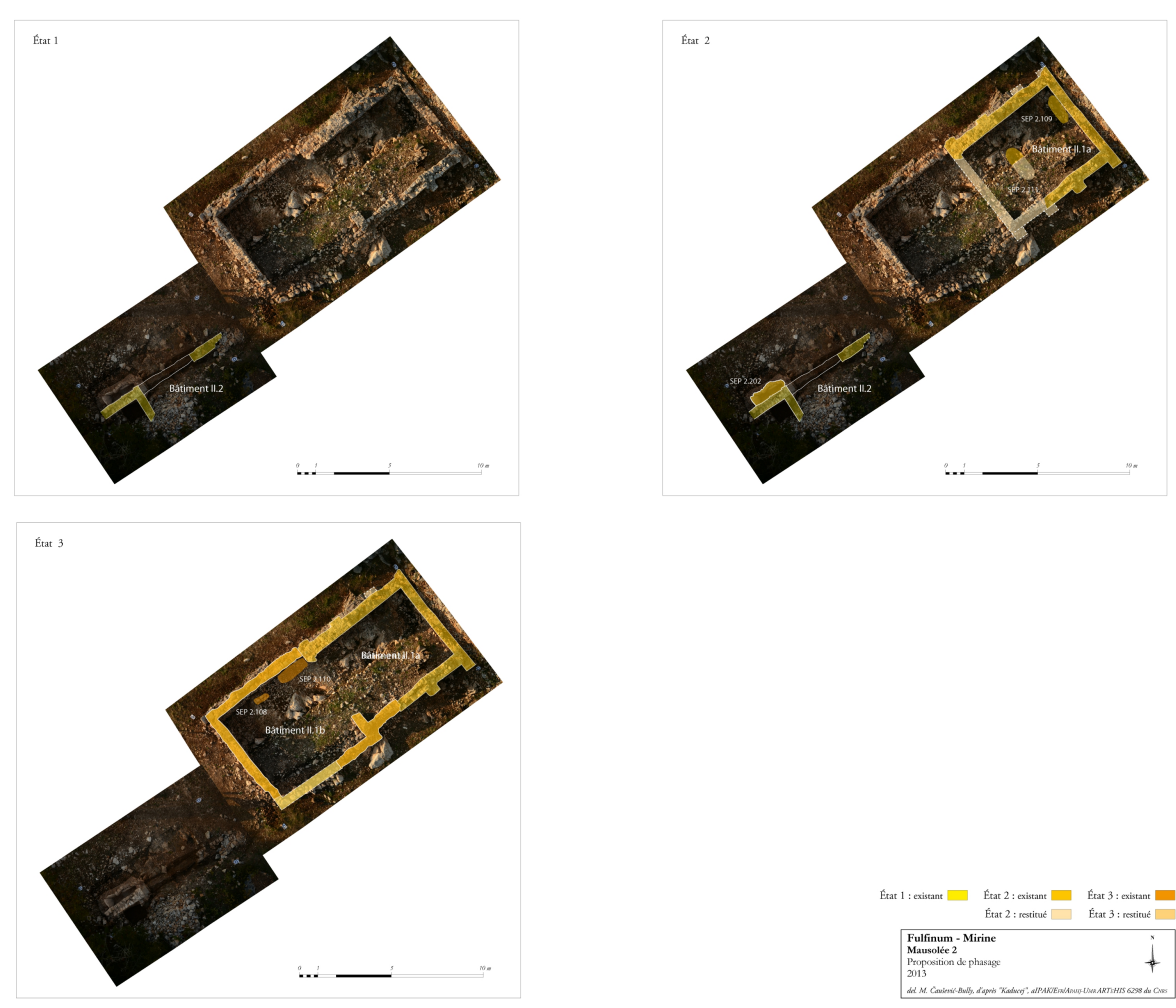

D’après M. Čaušević-Bully (infographie « Kaducej », Split).

65 Il suit une orientation respectueuse de la trame urbaine et périurbaine du site (nordest/sud-ouest). Les maçonneries en opus incertum, conservées sur un peu plus d'un mètre en élévation, mesurent $0,68 \mathrm{~m}$ d'épaisseur; les parements extérieurs sont scandés par une série de contreforts que l'on restitue au nombre de quatre par murs gouttereaux en fonction des traces d'arrachements et du rythme. La disproportion de la taille des contreforts $( \pm 0,68 \times 0,40 \mathrm{~m})$ par rapport aux dimensions du mausolée indique un espace voûté à l'origine.

Dans une seconde phase (II.1B), le mausolée est agrandi par l'adjonction en façade sud d'une seconde salle, plus large, de plan quadrangulaire de 5,5 x 5,6 m hors-œuvre. Les maçonneries en opus incertum de la seconde phase sont identiques en largeur, mais ne comportent pas de contreforts. L'espace devait être couvert par une simple charpente.

67 La fouille partielle du mausolée primitif a révélé deux tombes aménagées dans le rocher : une première (2.111) en position centrale, et une seconde le long du mur nord (2.109). Chacune contenait l'inhumation d'une femme immature d'après l'étude anthropologique. La femme inhumée au pied du mur nord était dotée d'une parure composée d'un bracelet en bronze sur son bras droit et de deux bracelets au bras gauche - un en fer et l'autre en bronze (fig. 18). 
Fig. 18 - Mirine-Fulfinum, mausolée, sépulture 2.109 avec mobilier funéraire.

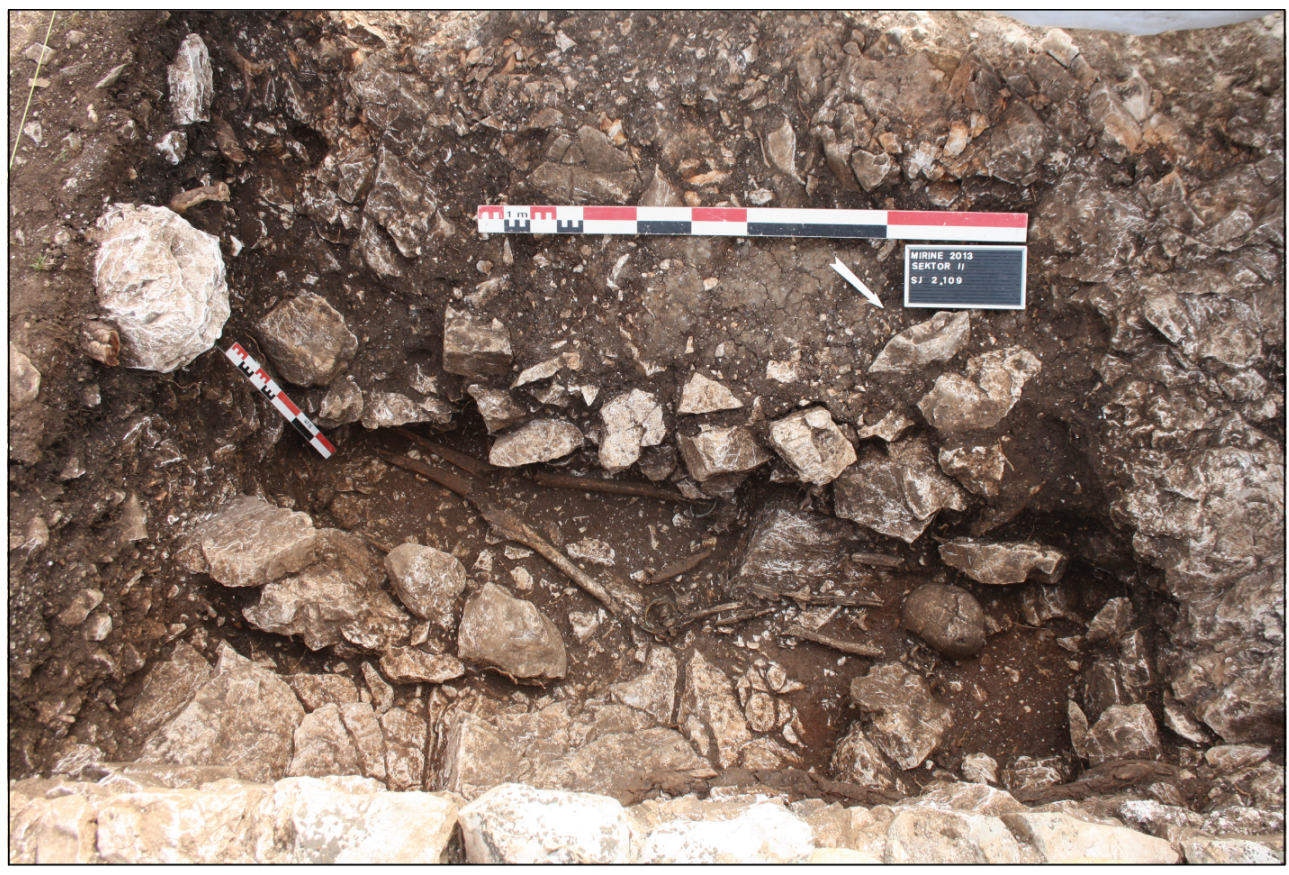

Cl. M. Čaušević-Bully.

Un couteau qui devait être initialement accroché à la ceinture a été découvert entre les jambes. Cette tombe est datée des premières décennies du $\mathrm{V}^{\mathrm{e}} \mathrm{s}$. par radiocarbone ${ }^{20}$. L'adjonction sud a livré une tombe (2.110) qui a été utilisée à deux reprises dans un lap de temps assez long, ce qui suppose un marquage de surface. Ce même espace a accueilli l'inhumation d'un bébé déposé dans une fosse en pleine terre pourvu d'un calage de pierres (2.108). Un caveau maçonné - non fouillé - est adossé contre le parement externe du mur nord (2.118).

Un second bâtiment (II.2) conservé sur une élévation d'environ 0,50 $\mathrm{m}$ a été identifié dans le prolongement sud du mur est du mausolée (2.105), mais sans que l'on puisse établir une chronologie relative entre les deux constructions en raison de l'état de conservation des vestiges. Le bâtiment B est reconnu par un segment de sa façade occidentale (2.201 et 2.203) ainsi que l'angle d'une pièce interne - à l'est - (2.200) couvert d'un sol de terrazzo (2.210). La façade est percée d'une large ouverture de 2,9 m (2.204) dont subsiste le calage d'un seuil monolithe disparu (fig. 19). 
Fig. 19 - Mirine-Fulfinum, seuil du bâtiment II.2.

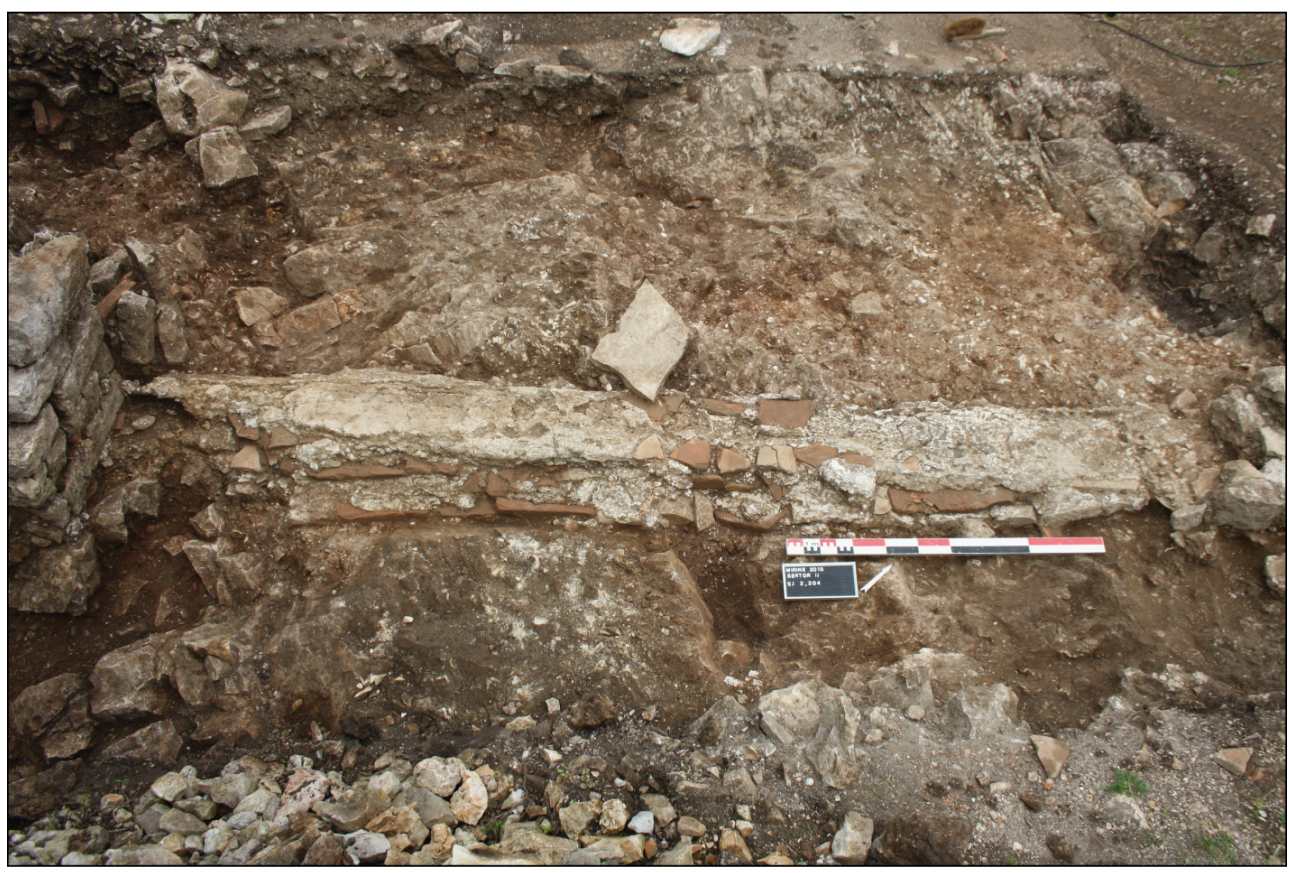

Cl. M. Čaušević-Bully.

70 Le mode de construction est soigné, avec un appareil régulier et assisé en parement extérieur, contrastant avec la maçonnerie fruste des deux phases du mausolée. Le mobilier recueillit dans ce secteur contient du verre à vitre, des restes de faune et des coquillages, de la céramique fine domestique de l'Antiquité tardive et des fragments d'amphores de type LRA.

71 Dans une phase postérieure, une tombe en coffre de dalles calcaires (2.202) est installée contre le parement extérieur de la façade (fig. 20). 
Fig. 20 - Mirine-Fulfinum, tombe à dalles 2.202 contre le bâtiment II.2.

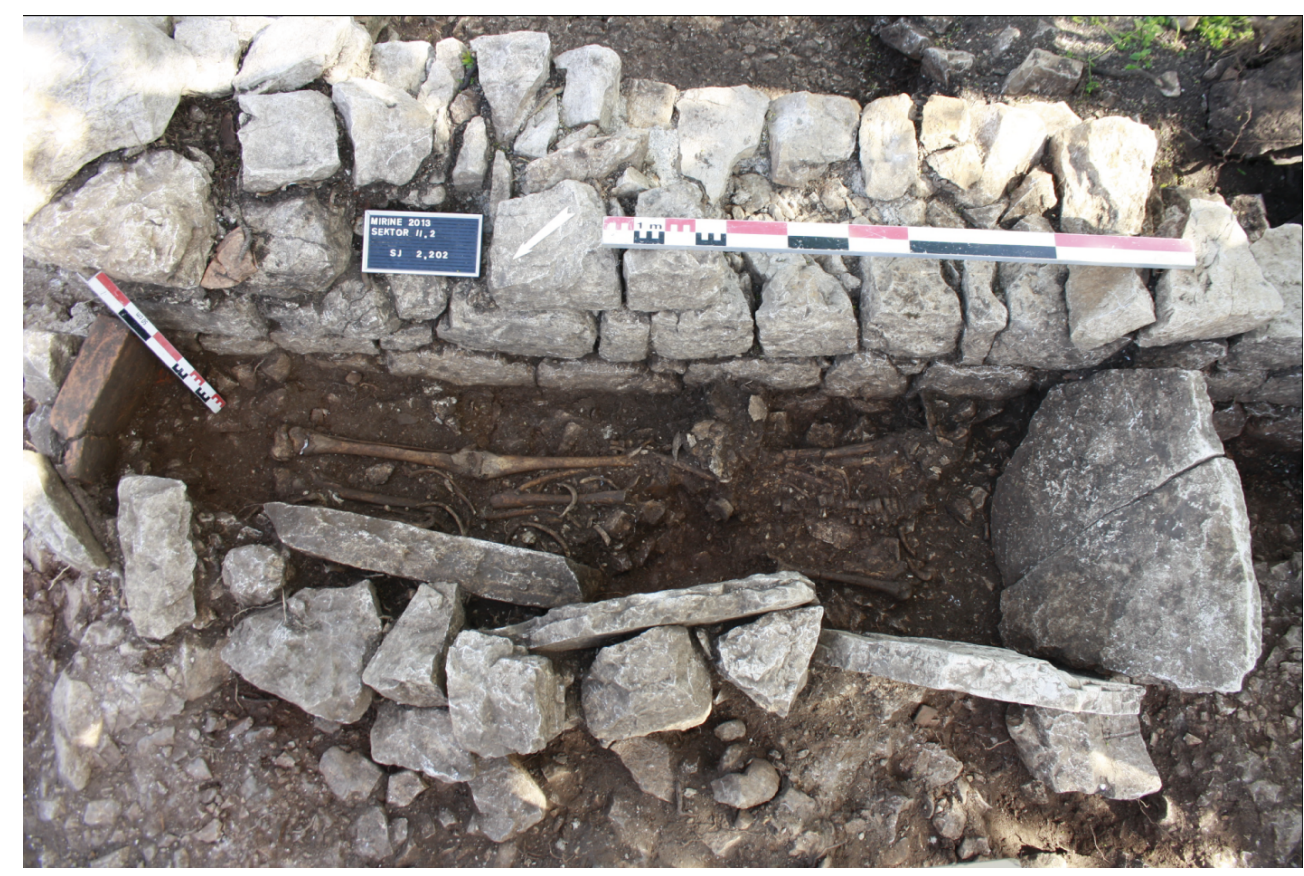

Cl. M. Čaušević-Bully.

72 Elle contient l'inhumation d'un adolescent de moins de 15 ans.

\section{Synthèse}

73 Si l'identification d'un mausolée du $V^{e}$ s., agrandi dans un second état, ne fait aucun doute pour le bâtiment II.1, celle du bâtiment II.2 reste indéterminée. Il pourrait s'agir d'une construction d'une certaine ampleur, en marge de la ville antique et en usage durant l'Antiquité tardive, mais sans que l'on puisse déterminer si elle est contemporaine du mausolée ou antérieure. La présence de la tombe en coffre de dalles contre la façade externe implique que le bâtiment pouvait avoir eu une fonction funéraire à un moment donné, ou que la nécropole s'est installée dans ses ruines... Seule une poursuite de la fouille permettra de répondre à cette question.

revanche, il est désormais fort peu envisageable que les constructions se développant à l'est $\mathrm{du}$ complexe paléochrétien aient appartenu au monastère, contrairement aux premières hypothèses. Cet ensemble participe donc à la connaissance de l'évolution du site de Fulfinum-Mirine durant l'Antiquité tardive avec l'installation sur les marges de la ville d'une nécropole au $\mathrm{IV}^{\mathrm{e}} \mathrm{s}$., rapidement dotée de mausolées et d'un important complexe paléochrétien dont la réutilisation monastique reste à préciser (fig. 21). 
Fig. 21 - Mirine-Fulfinum, vue générale du site entre le forum antique et le complexe paléochrétien.

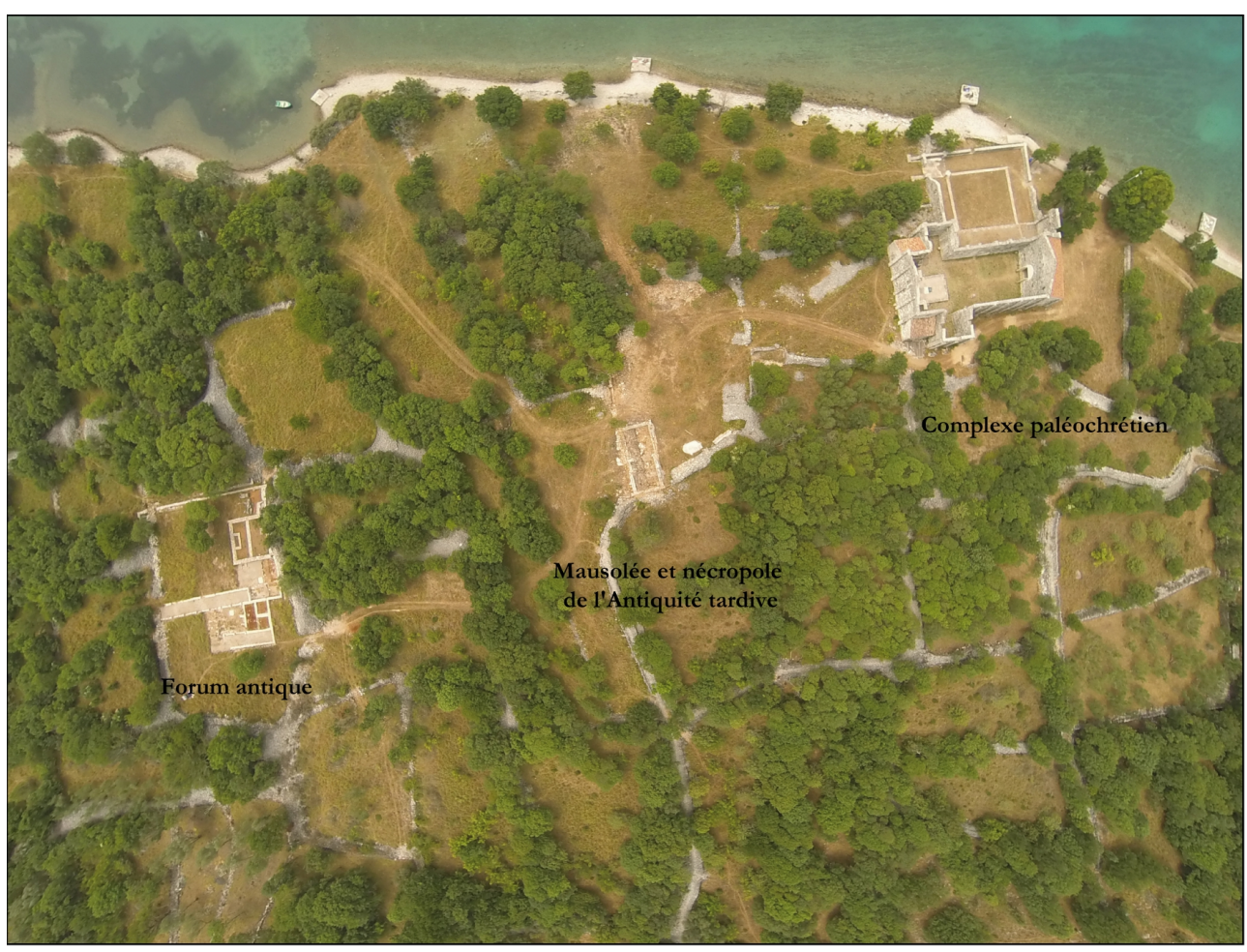

Cl. M. Vuković.

75 Rappelons seulement que les sépultures fouillées dans le vestibule de la basilique et dans les portiques de l'atrium attestent une utilisation au moins jusqu'au VIII ${ }^{\mathrm{e}} \mathrm{s}$. Il s'agit alors d'un recrutement exclusivement masculin qui pourrait correspondre à une communauté de clercs ou de moines.

\section{BIBLIOGRAPHIE}

Bully - Čaušević-Bully 2011 = S. Bully, M. Čaušević-Bully, Le monachisme insulaire dans l'archipel du Kvarner (Croatie) entre le $V^{e}$ et le XI ${ }^{e}$ siècle. Projet de recherches archéologiques, 2011-2014, dans Bulletin du centre d'études médiévales d'Auxerre (BUCEMA), 15, 2011, p. 335-350 http://cem.revues.org/ index12017.html.

Čaušević-Bully - Bully 2013 = M. Čaušević-Bully, S. Bully, Esquisse d'un paysage monastique insulaire dans le nord de l'Adriatique : l'archipel du Kvarner (Croatie), dans Hortus Artium Medievalium, 19, 2013, p. $167-182$

Chevalier 1995 = P. Chevalier, Salona II. Ecclesiae Dalmatiae : l'architecture paléochrétienne de la province romaine de Dalmatie (IV ${ }^{e}-V_{I}{ }^{e}$ s.) en dehors de la capitale, Split-Rome, 1995 (Collection de l'École française de Rome, 194/2). 
Mohorovičić 1957 = A. Mohorovičić, Problem tipološke klasifikacije objekata srednjovjekovne arhitekture na području Istre i Kvarnera, dans Ljetopis [JAZU], 62, 1957, p. 486-528.

Nedved 1990 = B. Nedved, Felix Arba - Sretan Rab. Pregled povijesti i spomenika otoka Raba u rano rimsko doba, Rab : SIZ za kulturu Općine Rab i Arheološki muzej u Zadru, 1990

Ostojić 1963 = I. Ostojić, Benediktinci u Hrvatskoj, vol. II, Split, 1963.

Rizner = M. Rizner, Konzervatorska studija otoka Raba, exemplaire d'auteur.

Ruffieux 2010 = Ph. Ruffieux, Céramique commune de l'Antiquité tardive découverte sur le site de l'église Sainte-Cécile à Guran, en Istrie, dans Hortus Artium Medievalium, 16, 2010, p. 247-266.

Skok 1952 = P. Skok, Slavenstvo i romanstvo na našim otocima, Zagreb, 1952 [JAZU].

\section{NOTES}

1. Bully - Čaušević-Bully 2011, p. 335-350 ; Čaušević-Bully - Bully 2013, p. 167-182.

2. Saint-Pierre sur l'îlot d'Ilovik, Martinšćica sur l'île de Cres, Saint-Jean sur l'îlot d'Oruda, l'îlot de Lukovac dans la baie de Crnika sur l'île de Rab, Saint-Laurent de Krk sur l'île de Krk, Mirine d'Omišalj sur l'île de Krk.

3. Le financement des recherches est assuré par le ministère des Affaires étrangères et européennes français, le ministère de la Culture croate, l'École française de Rome et la fondation Caritas Veritatis envers laquelle nous exprimons toute notre gratitude. Avec les auteurs de cet article, l'équipe archéologique était formée par Laurent Fiocchi, Brunilda Bregu, Josip Burmaz (société XXX) (relevés topographiques) ; de Tonka Kruzić (anthropologue), Ivan Valent, Thomas Chenal, Valentin Chevassu, Alicia Mougin, Pascale Chevalier, Ivona Belina, Iva Vezmar, Miro Vuković (couverture photographique par drone), Luka Babić (assistant de recherche, université de Zagreb, scanner 3D). L'essentiel de la gestion et de la logistique de l'opération est géré par les structures aIPAK et APAHJ.

4. Chevalier 1995, p. 60-62.

5. La pièce L3 n'a pas été fouillée.

6. Le verre à vitre et la gobeleterie sont en cours d'étude par Inès Pactat, doctorante à l'université de Franche-Comté.

7. L'étude des monnaies a été réalisée par Zrinka Ettinger Starčević, directrice du Musée de Mali Lošinj.

8. Ruffieux 2010, p. 247-266.

9. En remerciant Stefano Zucchi pour cette information.

10. Ostojić 1963, p. 157-158.

11. Bully - Čaušević-Bully 2011.

12. Mohorovičić 1957 , p. 486-528.

13. Ibid.

14. Relevés réalisés par Luka Babić, université de géodésie de Zagreb.

15. Ce toponyme est vraisemblable car on trouve une grande quantité de pieds d'oignon sauvage sur l'îlot.

16. Rizner ; Nedved 1990.

17. Les contacts avec les autorités locales ont été rendus possibles par Mia Rizner, du Centre de préservation des Monuments de Rijeka, que nous tenons à remercier.

18. Ruffieux 2010.

19. Skok 1952.

20. Analyse Beta-359177 ; 68\% de probabilité : 400-430 AD avec un pic de probabilité en 420 . 
INDEX

Index géographique : Croatie, Mirine, Martinšćica, Kvarner institutions ARTeHIS UMR 6298 du CNRS (Dijon-Auxerre), École française de Rome

Mots-clés : insularité, archéologie du bâti, monastère, église, mausolée, funéraire, villa

\section{AUTEURS}

MORANA ČAUŠEVIĆ-BULLY

École française de Rome - morana.causevic[at]gmail.com

SÉBASTIEN BULLY

CNRS, UMR 6298 ArTeHiS - sebastien.bully[at]club-internet.fr 